\title{
An Estimation Theoretic Perspective on Image Processing and the Calculation of Optical Flow
}

\author{
T.M. Chin \\ RSMAS. L'. of Miami. Mliami FL. US.A \\ M.R. Luettgen \\ M.I.T.. Cambridge MA. USA \\ W.C. Karl \\ M.I.T. ('ambridge MA. ISA \\ A.S. Willsky \\ M.I.T. ('ambridge MA. CIS.A
}

\subsection{Introduction}

Yany problems of image processing and image sequence analysis involve both ureal computational complexity and the accommodation of noise and uncerlainty through the indirect observation of quantities of interest. In this chapter "Wr describe several aspects of an estimation theoretic approach to such probloms. The vehicle for our development is the estimation of the apparent velocity licld of a sequence of images. This apparent velocity field. known as the optical IInw. appears as an important quantity in both the qualitative and quantitative allalysis of image sequences. For example. knowledge of the optical flow is used in lhr letection of object boundaries and the segmentation of visual scenes [1.2] the lerivation of $3-D$ motion and structure $[3,4]$, and the compression of image menences for efficient transmission [5. 6].

We rake an estimation-theoretic perspective to the computation of optical 
flow. using and extending the formulation of Rougee et al. [i. 8$]$ in both the temporal and spatial directions. In particular. we use model-based interpretations of the various components arising in the estimation theoretic setting to allow us to develop novel extensions to existing approaches. First, we consider the imposition of a trmpornl coherence to the flow obtained by modeling the evolution of the vector optical flow process with a linear state equation and then applying a recursive Kalman filter to the observalions obtained from the image sequence. The classical (IIorn and Schunk [9]) formulation of the optical flow estimation problem contains no such formal requirement of temporal colicrence. The inciusion of such a constraint allows the reliable and robust cstimation of optical flow under conditions difficuit for the classical approach. Lor example. in situations where a single image pair contains insufficient information to recover the flow field due in the "aperture problem." the integration of observations nuer a longer time frame can yield reasonable results.

Applications of Kalman filtering to various formulations of optical flow estimation $[10.11$ ) as well as to other low-level reconstruction problems in computational vision $[i 2$ ! have been proposed. In these previous approaches. however. the apparently computationally daunting task of implementing the Kalman filtering equations. and in particular the error covariance equations. on even moderat elysized images resulted in the use of drastically simplified and suboptimal filter specifications. Specifically, the uncertainty in the dynamic model for the timevarying unknown field. and hence the uncertainty in the estimate itself. is not formally represented or properiy propagated in these approaches. In an exact implementation of a lialman filter. such uncertainty. as captured in the estimation error covariance matrix, is propagated along with the estimate itself [13. 14. 15] and allows for the optimal fusing of the current estimate with new observations. The filtering algorithm presented in this paper cmploys a more systematic and rational approximation of the Kalman filter than those previously reported. This approximation is based on the propagation of approximate local models of the estimation error covariance. These results provide. 10 curr knowledge. the first implementation of the romplete halman filtering equations for space-time problems of this scale. and the only example of successful. near optimal. propagation of covariance matrices of this size. The mathematical details of our approximation techniques can be found in [16] in the more general context of low-level visual reconstruction.

Second. we use the observation that both the single and multi-frame problems can be formulated as spatial estimation problems. wherein sets of observations are fused with prior spatial field models. to motivate the use of a recently dereloped class of multiscale statistical models in their solution. What makes these multiscale field models especially interesting is 1) that there exist extremely officient. multigrid-type estimation algorithms based on them and 2) that a large number of degrees of freedom exist in their specification. allowing them to approximate a wide range of different flows. including. as least conceptually. any Markov Random Field based flow. Together. these qualities imply that the utilization of such multiscale spatial models for spatial estimation prohlems. and in particular for the optical flow problem. provides a flexible cet retromrly of- 
licient estimation framework. Preliminary examples of our results are provided showing factors of $10-100$ computational improvment over conventinnal methwis. Finally. such models provide multiscale representations of the flow field and. though we have not used it here. also provide the possiblility of optimal int.cgration of multiscale measurements.

In this chapter we focus on a particular image processing problem. namely lhe computation of optical flow. However. the model-based approaches used lirere are more generally applicable to the wide range of space-time estimation problems arising in image sequence processing.

\subsection{Optical Flow Estimation}

\subsubsection{Single-Frame Formulation}

Ihe 2-1) vector field of the apparent motion of brightness patterns in an image is werred to as the optical flow ! 9$]$. One conmonly used way to obtain informatimn about the optical flow field $x\left(z_{1} . z_{2} \cdot t\right) \equiv\left[d z_{1} / d t \text {. } d z_{2} / d t\right]^{T}$ at a given point in space $(z, 2, z)$ and time $t$ was presented by Horn and Schunck in [9]. This ipproach is based on the assumption that. changes in scene brightness in the image seffuence are due only to motion. This assumption leads to the so called lirighturs.se ronstrnint fquation [?]:

$$
0=\frac{d}{d t} E\left(z_{1} \cdot z_{2} \cdot t\right)=\frac{\partial}{\partial t} E\left(z_{1} . z_{2} \cdot t\right)+\Gamma E\left(z_{1} . z_{2}, t\right) \cdot x\left(z_{1} . z_{2}, t\right)
$$

where $E\left(=1, z_{2}, t\right)$ is the image intensity as a function of time and space and $\left.\Gamma l:=[\partial E / \partial) \Sigma_{1} . \partial E / \partial z_{2}\right]$, is the gradient of the image intensity.

The brightness constraint equation ( 2.1 ) does not completely specify the flow lirld since it provides only one linear constraint for the two unknown components if $x\left(z=z_{2} .1\right)$ at each point. This is usually referred to as the aperture problem [!]. () we way to obtain a unique solution is to regularize the problem by imposing in additional smoothness constrmut. Specifically. une lormulates the following aprimization problem [?]:

$$
\underset{x\left(1,1, z_{2}, t\right)}{\operatorname{argmin}} \iint \nu\left\|\frac{\partial}{\partial t} E\left(z_{1}, z_{2}, t\right)+\Gamma E\left(z_{1}, z_{2}, t\right) \cdot x\right\|^{2}+\|\Gamma x\|^{2} d z_{1} d z_{2}
$$

The smoothness constraint is captured by the second term which penalizes large wradients in the optical flow and is necessary to make the formulation mathemantically well-posed [17]. This term also represents our prior belicf about the llow fichd. implying that the computed flow should vary smoothly over space. Sinch spatinl roherence of the flow vectors reflects the smoothness and stiffuess if the ohject surface in the scene [18]. The constant $\nu\left(z_{1} . z_{2} . t\right)$ allows one to 1 radcoff between the relative inportance in the cost function of the brightness and smoothness constraint terms.

Before proceeding let us analyze the smoothness constraint in more detail. Vote that the penalty associated with the smoothness constraint term in $(2.2)$ is cplual to the integral of the squared norm of the field gradient over the image 

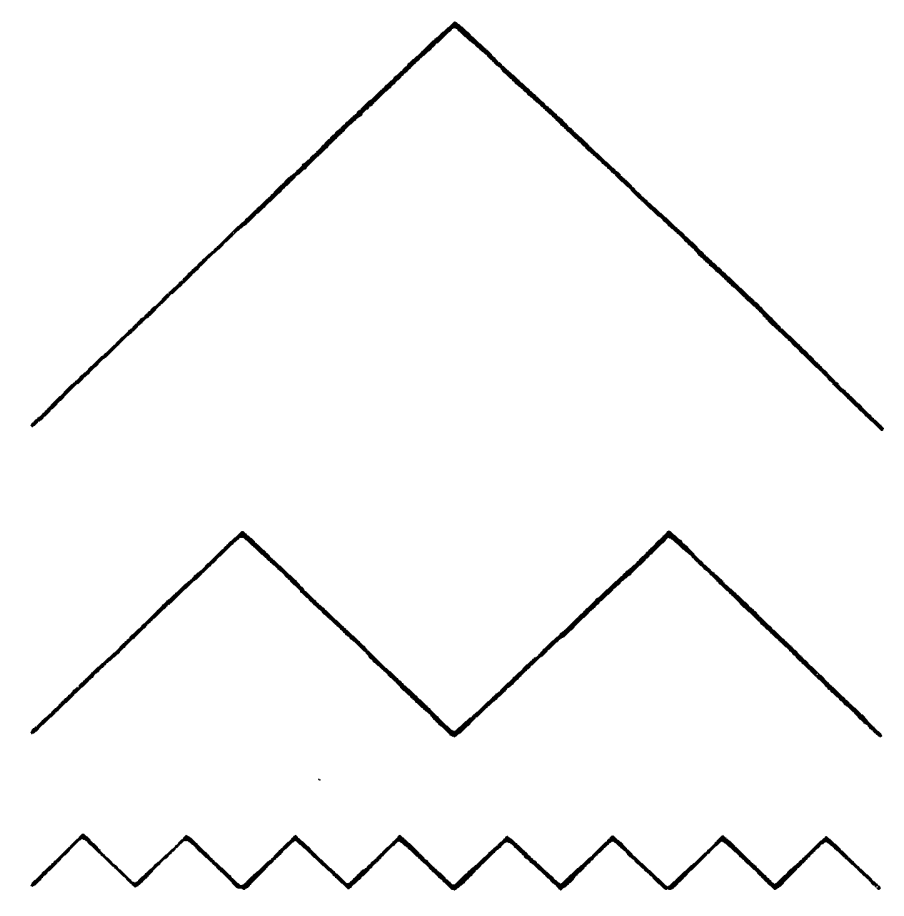

Figure 2.1: Depiction of three fields which are equally favored by the smoothntiss constraint. illustrating how this penalty provides a fractal prior modef for the optical flow.

plane. In a one-dimensional context. such a constraint would penalize each of the (one-dimensional) fielels in Figure 2.1 equally. Intuitively. the smoothness constraint has a fractal nature. and in fact this can be demonstrated in a much more precise sense, as we show in Section 2.3.1.

\subsubsection{Multi-Frame Formulation}

The formulation $(2.2)$ processes the data (i.e. the gradients of the image intonsity) a frame at a time. vielding flow estimates independently over time. The imposition of temporal coherence [19] to the flow field can be considered in addition to the spatial coherence enforced by $(2.2)$ in order to utilize more data for each flow vector estimate. Temporal coherence imposes an inertia condition on the flow field. favoring gradual changes in the optical flow vectors over time. Temporal coherence models of optical flow are applicable to a wide range of motions in uatural scenes. as most movements display inertia of some type. To obtain such a multi-frame formulation of the optical flow computation problem we use a simple temporal extension of $(2.2)[20.12]$. In particular. We find the flow field $\hat{x}\left(z_{1}, z_{2}, \tau\right)$ which is the solution to the following problem at time $t=r$ :

$$
\underset{x\left(z_{1}, z_{2}, t\right)}{\operatorname{argmin}} \int_{0}^{T} \iint_{D} \nu\left\|\frac{\partial E}{\partial t}+\Gamma E\left(z_{1}, z_{2}, t\right) \cdot x\right\|^{2}+\|\Gamma \cdot x\|^{2}+\rho\left\|\frac{\partial}{\partial t} \cdot\right\|^{2} d . s d t . \quad(2 . .3)
$$

The multi-frame formulation $(2.3)$ is obtained from the single-frame formulation (2.2) by the addition of a quadratic term involving the first order temporal 
Irrivative. Note that the full solution to the optimization problem $(2.3)$ leads Io a. reconst.rneted space-time ficld $\widehat{x}\left(z=z_{2}, t\right)$ in which the reconstruction at. any time takes advantage of all available constraints over the entire time interval $0 \leq t \leq \tau$. We are only interested in the value of the solution to $(2.3)$ at the cirrent time $t=r$, corresponding to the best causal or filtered estimate of the llow field. Filtered estimates are desirable in applications where the optical flow nerds 10 be calculated as soon as each frame in the image sequence becomes available: however, obtaining such estimates corresponds to solving a different 3-1) optimization problem for each $T$ as $\tau$ increases. Such a solution clearly results in a greatly increased computational burden over what is required for the single-frame solution of $(2.2)$. making direct solution of the optimization problem (2.3) prohibitive.

\subsection{Discretization and Probabilistic Interpretation}

II the first part of this section we present a discrete formulation of the singlelammo aptical how problem. In this context we develop two model-bnsfod intermratitions of the single-frame problem which will be central to the results of hertions 2.4 and 2.5. In particular. we illustrate 1) how the smoothness conhrainl can be interpreted as a prior probabilistic spatial model for the flow field and 2) how the inverse of the covariance of the field estimate may be naturally inlerprencl as another spatial model. this time for the postcrior estimation crror "f thr field. The second part of the section shows how we may model the temporal roherence constraint of (2.3) by a discrete dynamic equation. which may then hw conpled with a set of observation equations obtained from the single-frame case to vield an equivalent state estimation problem.

\subsubsection{Single-Frame Case}

In practice. brightness measurements are only available over a discrete sot of prints in space and time. Thus. the temporal and spatial derivative terms must he approximated with finite differences. and the optical flow is only estimated on a discrete space-time grid. There are a number of important issues which arise din in the discretization which we do not discuss here: we refer the reader to [21] for a detailed treatment. We will assume here that we have normalized the space-lime coordinates so that the optical flow is to be estimated on the set of inlongers $\left(z_{1}, z_{2}\right) \in\left\{(i, j) \mid i, j \in\left\{1, \cdots, 2^{M}\right\}\right\}$. where $M$ is also an integer, so the lolal number of image points is $\lambda \equiv 4^{M}$. The assumption that the grid is square and that the number of rows is equal to a power of two makes the derelopmont uf Sirction 2.5 easier. but is not essential. Now at grid point $(i . j)$ at time $t$ In. us define $y(i, j) \equiv(-\partial E(i, j, t) / \partial t)$ to be the measured temporal brightness drrivative. $r(i . j)$ to be the desired optical flow vector. and $C^{\prime}(i . j)=\Gamma E(i, j . t)$ to bo the spatial gradient of the image brightness (also measured). Then we may write the brightness constraint $(2.1)$ at the point $(i . j)$ at time $t$ as:

$$
y(i . j)=C(i . j) x(i . j)
$$


The brightness constraints $(2.4)$ at all grid points can now be grouped into one large set of linear equations to concisely capture the optical flow information contained in the image sequence. Let $\mathbf{x}(t)$ be a vector containing the optic s ow values $x(i, j)$ at all the grid points at time $t$ ( using, say, a lexicographic orci and $\mathbf{y}(t)$ be the associated vector of the samples $y(i, j)$. Similarly. let $\mathbf{C}$ ind $\mathbf{W}(t)$ be block diagonal matrices whose diagonal elements are the samples $i . j)$ and $\nu(i, j)$, respectively, taken in the same order at time $t$. A discrete rsion of the single-frame formulation $(2.2)$ is then given by:

$$
\hat{\mathbf{x}} s C^{(}(t)=\underset{\mathbf{x}(t)}{\operatorname{argmin}}\left\{\|\mathbf{y}(t)-\mathbf{C}(t) \mathbf{x}(t)\|_{\mathbf{W}(t)}^{2}+\|\mathbf{S} \mathbf{x}(t)\|_{I}^{2}\right\}
$$

where $\|\mathbf{x}\|_{\mathbf{W}}^{2}$ denotes the weighted norm $\mathbf{x}^{T} \mathbf{W} \mathbf{x} . I$ represents the identity matrix, and $S$ is the matrix first-order spatial difference operator. Note that the discrete nature of the problem alluded to above implies that we must actually approximate the samples $-\partial E(i, j, t) / \partial t$ and $\Gamma E(i . j, t)$. and thus $\mathbf{C}$ and $\mathbf{y}$ by finite differences. The spatially varying entries of $W$ can actually be used to reflect our confidence in these approximations. We refer the reader $10[21]$ for further details.

\section{Spatial Models}

An estimation-theoretic formulation of the optimization problem in (2.5) ran now be developed. and we will use it to show that the statistirally optimal estimate of the optical flow, given a particular set of measurements. is identical to the smoothness constraint solution given in (2.5). Specifically, solving the quadratic minimization problem $(2.5)$ is equivalent to solving a maximum likelihood ( $M L)$ estimation problem [1.5] for $\mathbf{x}(t)$ with the following obsfrration equations:

$$
\begin{aligned}
\mathbf{y}(t) & =\mathrm{C}(t) \mathbf{x}(t)+\mathbf{r}_{1}(t) \quad \mathbf{r}_{1}(t) \sim\left(0 . \mathbf{W}^{-1}(t)\right) \\
0 & =\mathrm{S} \mathbf{x}(t)+\mathbf{r}_{2}(t) \quad \mathbf{r}_{2}(t) \sim(\mathbf{0 . I})
\end{aligned}
$$

where we have used the notation $\mathrm{x} \sim(\mathrm{m} . \mathrm{H})$ to denote a (iaussian random vector $\mathbf{x}$ whose mean and covariance are $\mathbf{m}$ and $\mathbf{H}$. respectively, so $\mathbf{r}(t) \equiv\left[\mathbf{r}_{1}(t) . \mathbf{r}_{2}(t)\right]^{T}$ is a zero-mean Ciaussian random noise process. Thus. the maximum likelihood problem formulation results in the same solution as the smoothness constraint formulation when $S$ is used to define an additional set of noisy measurements.

By formulating the problem in this estimation-theoretic framework. we can use $(2.7)$ to interpret the smoothness constraint as a prior probabilistic spatial model for the flow field. Specifically, we can rewrite $(2 . T)$ as:

$$
\mathbf{S x}(t)=-\mathbf{r}_{2}(t)
$$

Recalling that $S$ is an approximation to the gradient operator. We see that $(2.8)$ is nothing more than a spatial difference equation model for $\mathrm{x}(t)$ driven $b y$ the spatial white noise field $\mathbf{r}_{2}(t)$. In particular. this prior model represents the optical flow field as composed of independent. two-dimensional Brownian 
motions ${ }^{1}[\bar{\tau}, 8]$. Then. the statistically optimal estimate of the flow field. given lho measurements (2.6) and the Brownian motion prior model. is the same as the opt.ical flow estimate given by (2.5). The estimation-theoretic interpretation simply allows us to interpret the smoothness constraint as a Brownian motion moricl. In one-dimension. Brownian motion is a statistically self-similar. fractal process with a $1 / f^{2}$ generalized spectrum [22]. and for this reason the smoothness constraint is often referred to as a "fractal prior" [12]. We will return to this interpretation in Section 2.5 where we discuss a multiscale modeling approach to the single frame problem. In particular. we will replace the prior model $(2.8)$ ly a similar but multiscale prior model, which leads to dramatic computational arings.

Next. let us consider another model based interpretation of the single-frame problem (2.5) that will be useful in treating the multi-frame problem. The ML istimate for the optical flow. $\hat{x}(t)$, based on the measurements $(2.6) \cdot(2 . \overline{)})$ is whtaincel as the solution of the following inverse problem:

$$
\left(\mathbf{C}^{T}(t) \mathbf{W}(t) \mathbf{C}(t)+\mathbf{S}^{T} \mathbf{S}\right) \hat{\mathbf{x}}(t)=\mathbf{C}^{T}(t) \mathbf{W}(t) \mathbf{y}(t)
$$

The mplations in (2.9) represent a discrete version of the coupled Poisson equalinns of the Ilorn and Schunck formulation. The matrix operator

$$
\left.\mathrm{L}(t)=1 \mathrm{C}^{T}(t) \mathrm{W}(t) \mathrm{C}(t)+\mathrm{S}^{T} \mathrm{~S}\right)
$$

(III Hhe Inft hand side of (2.9) has a sparse. nearest neighbor (a nested block 1 ri-diagunal) structure [23]. whose sparseness enables us to use efficient iterative procediures. such as multigrid methods [24]. in the solution of (2.9). Also. this sparse matrix corresponds to the information matrix (the inverse of the covariance matrix) associated with the posterior estimation error $\mathbf{d}(t) \equiv \mathbf{x}(t)-\hat{\mathbf{x}}(t)$. In particular $L(t)$ can naturally be considered to specify an implicit Markov Random Field model for the estimation error process $d(t)$ of the following form:

$$
\mathrm{L}(t) \mathrm{d}(t)=i(t) . \quad \zeta(t) \sim(\mathbf{0 .} \mathrm{L}(t))
$$

The nearest neighbor structure of $\mathrm{L}(t)$ in $(2.11)$ or $(2.9)$ reflects a corresponding linat structure to the statistical model for the estimated field error covariance. IV will use this observation in Section 2.4 to develop tractable yet near optimal lilloring algorithms.

\subsubsection{Multi-Frame Case}

Vow wn consider the multi-frame extension of the single-frame formulation given in (2.(j).(2.7). The continuons optimizalion problem $(2.3)$ can be considered to lo : 11 "ptimal smoothing problem based on the following temporal. linear (ianssMarkor dynamic system for $\mathbf{x}(t)[16]$ :

$$
\frac{\partial}{\partial t} r\left(z_{1} . z_{2} \cdot t\right)=q(t)
$$

\footnotetext{
'More precisely. to avoid biasing the optical flow estimates towards zero. we only assumc that the qradients of the optical flow field components are equal to the gradients of the Brownian motion processes. This avoids placing a constraint on the DC' (i.e. average) value of the optical ll.w and formses nuly on imposing a : refernme for smoothness in the flow.
} 
where $q(t)$ is a Gaussian white noise process of zero mean and intensity $\rho^{-1}$. For such an optimal smoothing problem. two-filter methods (i.e. obtained by running a Kalman filter in each of the causal and anti-causal directions) are applicable [i]. In general we wish to compute only the most recent estimate $\hat{\mathbf{x}}\left(z_{1}, z_{2}, \tau\right)$ from (2.3) for each $r \geq 0$. Such an estimate can be obtained by a single causal Kalman filter. Specifically, a discrete version of this multi-frame problem can be formulated as a state estimation problem for the dynamic system whose dynamic equation is

$$
\mathbf{x}(t)=\mathbf{x}(t-1)+\mathbf{q}(t), \quad \mathbf{q}(t) \sim\left(\mathbf{0}, \rho^{-1} I\right)
$$

coupled with the observations given by $(2.6),(2.7)$. The process noise $q(t)$ is $111 \mathrm{l}-$ correlated over time and captures the uncertainty in the dynamic model (2.13). This Ciauss-Markov dynamic model. a discrete version of $(2.12)$, indicates that the optical How evolves in time as the accumulation of a random perturbation at each time frame. While we will be concerned with temporal dynamics of the form (2.13). naturally more complicated dynamic models. corresponding to different temporal coherence terms in $(2.3)$. could be used.

\subsection{Sequential Multi-Frame Estimation}

In this section we consider state estimation for the dynamic system represented by (2.1:3).(2.6),(2.7). Conceptually, we may use well-developed optimal sequential estimation algorithms, such as the Kalman filter and its variants. for solution of this multi-frame optical flow estimation problem. One such algorithm. that. will prove convenient for us, is the following implementation of the information form [13. 1.5] of the Kalman filter [16]:

- prediction stage

$$
\begin{aligned}
& \overline{\mathrm{L}}(t)=\rho I-\rho^{2}(\hat{\mathrm{L}}(t-1)+\rho I)^{-1} \\
& \overline{\mathbf{x}}(t)=\hat{\mathbf{x}}(t-1) \\
& \overline{\mathbf{z}}(t)=\overline{\mathrm{L}}(t) \overline{\mathbf{x}}(t)
\end{aligned}
$$

- update stage

$$
\begin{aligned}
\hat{\mathbf{L}}(t) & =\overline{\mathbf{L}}(t)+\mathbf{C}^{T}(t) \mathbf{W}(t) \mathbf{C}(t)+\mathbf{S}^{T} \mathbf{S} \\
\hat{\mathbf{z}}(t) & =\overline{\mathbf{z}}(t)+\mathbf{C}^{T}(t) \mathbf{W}(t) \mathbf{y}(t) \\
\hat{\mathbf{L}}(t) \hat{\mathbf{x}}(t) & =\hat{\mathbf{z}}(t)
\end{aligned}
$$

where $\overline{\mathbf{x}}(t)$ is the one-step predicted estimate and $\hat{\mathbf{x}}(t)$ is the updated esimate using the new data available at time $t$. Also. $\overline{\mathrm{L}}(t)$ and $\hat{\mathrm{L}}(t)$ denote the predicted and updated information matrices. respectively. Note that the updated estimate $\hat{\mathbf{x}}(t)$ in $(2.19)$ is specified implicitly. as for the single-frame case (2.9). 


\subsubsection{Suboptimal Kalman filtering}

The number of pixels, $N$, in a frame of a typical image sequence is on the order if $10^{4}$ 10 $10^{6}$. Such a large number of points makes direct implementation of the iplimal information Kalman filter $(2.14)-(2.19)$ impractical as the associated information matrices $\overline{\mathrm{L}}(t)$ and $\hat{\mathrm{L}}(t)$ of the optimal filter will have on the order of $10^{*}$ io $10^{12}$ elements. The storage and manipulation of such large matrices is cinarly prohibitive. necessitating the use of a suboptimal method. The subiplimal filtering algorithm presented below employs a systematic and rational approximation of Kalman filter. which is based on the propagation of approximalc tocal models of the estimation error covariance. as discussed in connection wilh $(2.11)$.

To develop our sub-optimal filter. consider the set of equations $(2.1+)-(2.19)$. First consider the update stage of the Kialman filter. If $\bar{L}(t)$ possesses a sparse and handed nearest neighbor structure. as was true for the single-frame probinit. hell $(2.17)$ will preserve this structure in $\hat{L}(t)$ since. as we pointed out in connertion with $(2.9)$. $\mathbf{C}^{T}(t) \mathrm{W}(t) \mathrm{C}(t)+\mathrm{S}^{T} \mathrm{~S}$ also possesses this structure. In parricular. if this is the case. then $(2.19)$ may still be solved efficiently for the uprated estimate $\hat{\mathbf{x}}(t)$. and in fact this step would have fartly the same compulational complexity as in the single-frame case. Thus. we desire to preserve snch a sparse and banded structure in $\overline{\mathrm{L}}(t)$.

Now consider the prediction stage. Unfortunately. even if $\hat{\mathbf{L}}(t-1)$ in $(2.11)$ is initially sparse and banded. the predicted information matrix $\overline{\mathrm{L}}(t)$ will not be dur to the matrix inverse on the right hand side of this equation. In addition. linding the inverse of this matrix is a prohibitively complex procedure. What in insire in the present framework. then. is a sparse and banded approximation in $\overline{\mathrm{L}}(1)$ that may be efficiently computed.

Is totailed in [16, 21], such an approximation may incleed be obtained hy "xpanding the matrix inverse on the right hand of $(2.14)$ in a series as follows:

$$
\overline{\mathrm{L}}(t)=\rho I-\rho^{2}\left(\Omega^{-1}-\Omega^{-1} \Delta \Omega^{-1}+\Omega^{-1} \Delta \Omega^{-1} \Delta \Omega^{-1}-\cdots\right)
$$

ulwen! is a block diagonal matrix whose $2 \times 2$ diagonal blocks are identical to l he corresponding diagonal blocks of the matrix $\hat{\mathrm{L}}(t-1)+\rho I$ while $\lambda=\hat{\mathrm{L}}(t-$ $1)+\rho I-\Omega$ is given by the remaining off-diagonal part of $\hat{\mathrm{L}}(t-1)+\rho I$. Note that $\Omega^{-1}$ is block diagonal. The series (2.20) may now be truncated to any desired nIImber of terms to obtain an approximation to the exact expression of the Irsired level of accuracy. The more terms are kept. the less sparse and banded llic npproximation will become. Thus. there is a tradeoff between accuracy and compunational efficiency. Our experience has shown that retaining only the first two lorms rields excellent results. In particular. We obtain our near-optimal liltar by replacing the optimal prediction step $(2.1 .1)$ by the following two-term iproximation:

$$
\overline{\mathrm{L}}(t)=\rho I-\rho^{2}\left(\Omega^{-1}-\Omega^{-1} \Delta \Omega^{-1}\right)
$$

l'nlike (2.1.1). the suboptimal prediction step $(2.21)$ does indeed preserve the Ansirnd nearest neighbor structure in the (approximated) information matrix $\overline{\mathrm{L}}(1)$. 
It can be verified straightforwardly that propagating the information matrix in the approximate filter as in $(2.17)$ and $(2.21)$ costs only $O(N)$ flops prr frame and has a local. modular computational structure suitable for par: el implementation. Throughout the filtering procedure. the approximated srmation matrices maintain the nearest neighbor structure and have only $O($ non-zero elements. Thus, the approximate filter has significant computatior and storage advantages over the optimal Kalman filter. which normally re ures $O\left(\mathrm{~N}^{2}\right)$ storage elements and $O\left(N^{3}\right)$ flops per frame of data.

A useful way to understand our approximation is provided by a a examination of the update stage of the Kalman filter. In this part of the filter we are fusing the information from the previous prediction stage. as captured by $\overline{\mathrm{L}}(t)$ and $\overline{\mathrm{z}}(t)$ (or equivalenty $\bar{x}(t)$ ), with the new observation. In particular. $\overline{\mathrm{L}}(t)$ can naturally be thought of as specifying a prior model for the error $e(t) \equiv \mathbf{x}(t)-\bar{x}(t)$ in the current estimate of the following form:

$$
\overline{\mathrm{L}}(t) \mathbf{e}(t)=\zeta(t), \quad \zeta(t) \sim(\mathbf{0} . \overline{\mathrm{L}}(t))
$$

which is just the counterpart of (2.11) for the dynamic problem. This modet is then combined with the new observation to produce the best estimate $\hat{e}(t)$ of this error. The updated estimate $\hat{\mathbf{x}}(t)$ in $(2.19)$ is then equal to $\overline{\mathbf{x}}(t)+\hat{\mathbf{e}}(t)$. The update stage is thus just a static spatial estimation problem. where (2.2:2) represents a prior model just before the inclusion of new data. That is. by writing the observation equations (2.(j). $(2 . T)$ concisely as $\mathbf{g}(t)=\mathrm{H}(t) \mathbf{x}(t)+\mathbf{r}(t)$. where $\mathbf{g}(t) \equiv\left[\mathbf{y}(t)^{T} \cdot 0^{T}\right]^{T} \cdot \mathbf{H}(t) \equiv\left[\mathbf{C}(t)^{T} \cdot \mathbf{S}(t)^{T}\right]^{T}$. and $\mathbf{r}=\left[\mathbf{r}_{1}^{T} \cdot \mathbf{r}_{2}^{T}\right]^{T}$, the estimate $\hat{\mathbf{e}}(t)$ can be obtained by solving the following static spatial estimation problem:

$$
\left[\begin{array}{c}
0 \\
\mathbf{g}(t)-\mathbf{H}(t) \overline{\mathbf{x}}(t)
\end{array}\right]=\left[\begin{array}{c}
\overline{\mathbf{L}}(t) \\
\mathbf{H}(t)
\end{array}\right] \mathbf{e}(t)+\left[\begin{array}{c}
-i(t) \\
\mathbf{r}(t)
\end{array}\right] .
$$

which is statistically equivalent to obtaining the updated estimate $\hat{x}(t)$ of the unknown $\mathbf{x}(t)$ given the prediction $\overline{\mathbf{x}}(t)$ and observation $\mathbf{g}(t)$. Since the implicit model is specified by $\bar{L}(t)$. our approximation of this matrix by a sparse matrix of the given nearest neighbor structure in $(2.21)$ corresponds naturally to the specification of an approximate. reduced-order model for the spatial error process. In particular. this approximation may be viewed as the imposition of a Markov Random Field structure of fixed spatial extent on the flow field estimation-error [16]. Our approximation thus has a rational basis in estimation-theoretic considerations.

\subsubsection{Numerical Experiments}

We demonstrate the beneficial effects of the temporal coherence constraint. formulated as the dynamic model (2.13), and the efficacy of our near-optimal filter for optical fiow estimation by numerical example in this section. Recall that. for images of realistic dimension. such as we consider here. exact implementation of the optimal Kalman filtering equations is impossible and thus we apply the temporal coherence constraint (2.13) via our suboptimal filter of Section 2.4.1. 
1 detailed comparison of the suboptimal and true optimal filters demonstrating lie near-nptimality of our approximation can be found in [16]. Here. a svnhictic image sequence of a moving brightness pattern is processed by various multi-frame and single-frame optical flow estimation methods. and the improvements gained by using the particular temporal coherence constraint (2.13). as implemented by the filter we presented in Section 2.4.1. are compared to the conventional methods. Specifically. the following two methods are considered:

- SF (Single Frame)

This method is a discrete version of the single-frame computational approach proposed by Horn and Schunck [9]. Each frame of optical flow is computed independently, i.e.. without any provision for temporal integralion of data. by solving the inversion problem $(2.9)$ for $\hat{\mathbf{x}}(t)$.

- TCS (Temporal C'oherence. Suboptinally computed)

This method is the suboptimal but computationally efficient version of the optimal Kalnan filter: as described in Section 2.4.1. the prediction step $(2.14)$ of the Kalman filter is approximated as $(2.21)$.

Variants of these methods arise in different computational environments. Specifically. lhe inversion steps (2.9) for SF and (2.19) for TCS can be implemented hy ane of the following computational procedures. leading to variations in the algorithms above:

- ic (iterative inversion. iterations to convergence) In practice. the inversion problems are solved iteratively. We use CiaussSiridel iterations in the experiments here. Needless to sav. this iterative solution will converge to the true solution in the limit.

- is (iterative inversion. single iteration)

II time sequential processing. it is natural to initialize the iterative inversion at time $t$ with the estimate obtained at time $t-1$. providing a rrasonably good estimate for time $t$ even before the first iteration. $B y$ slightly "updating" this initial guess with a single (or a small number of ) ( ianss-Seidel iteration!s) at the present time. a fairly accurate estimate of the flow field can emerge after continuing the process over several time lrames [9]. although such estimates are suboptimal in the statistical sense.

III Ihis section. each computational method is made explicit by the name of its main algorithm suffixed by the name of the variation. e.g.. TCS-ic. SF-is. etc. .1so. in cach experiment. the initial frame of optical flow estimate is computed idmically by the SF-ic method for every participating computational method in orrdre to highlight the differences in the temporal effects of each method.

The method SF-is deserves special attention. This method is the approach 10) multi-frame optical flow estimation suggested by Horn and Schunck in [9]. It performs only one Ciauss-Seiclel iteration for the inverse problem (2.9) at each $t$ lint usse the estimate from the previous frame. $\hat{\mathbf{x}}(t-1)$. to initialize the current iteration. Unlike the SF-ic method. therefore. this method does have some i)ovi in for propagating the ostmates temporally. Sote that if. instead of onl: 
a single Ciauss-Seidel step, the iterations are allowed to converge for each frame of data. the resulting flow estimates would have lost all information from the previous frame and become exactly the same as the SF-ic estimates. Althongh the SF-is method is ad hoc in terms of its temporal integration of data. its ease in implementation is appealing from a practical point of view.

One of the advantages of using a temporal coherence constraint in optical flow estimation is the improvement in the estimates due to the reduced effect of measurement noise through the averaging of the noisy data over time. Another. less obvious advantage. is the temporal accumulation of complementary information regarding the flow vectors. Reconstruction of optical flow using only spatial data integration (i.e., the SF-is method) cannot be performed correctly when a complete set of the information necessary to estimate the flow vectors is not contained in each data frame. Specifically. since diversity in the orientations of the measured spatial gradients is necessary to resolve the aperture probicm. optical llow computation methods emploving only a spatial coherence constraint will have difficulties dealing with cases where all the spatial gradients happen to be oriented in nearly the same direction (including the cases where most of the spatial gradient vectors have small magnitudes). Ldclition of a temporal coherence constraint can often relieve such difficulties by allowing the use of information from adjacent image frames. The example we give below demonstrates both the fact that the temporal constraint is instrumental in correctly estimating the flow in such cases and that it aids in noise suppression.

\section{Stagnation Flow Experiment}

In this experiment we consider estimation of the motion of a non-rigid body using the SF-ic and TCS-ic methods as well as the SF-is and TCS-is methods.

1. The image sfquence.

Figure 2.2 shows a flow pattern whose velocity vector at point $(:=1.2)$ is given $h y\left(n s_{1},-n s_{2}\right)$ for $n=0.1$. where the coordinate origin is at the midpoint of the bottom edge of the figure. This type of flow thin an arbitrary constant $n$ ) is useful for a local characterization of stammotmun flow [25]. i.e. the flow of huid obstructed perpendicularly by a solicl ob jont. A sequence of $64 \times 48$ images are sinthesized based on such a velocity field. Figure 2.3 presents four images from the sequence. Note that the direction of the predominant contrasts in each image changes from mostly vertical in the early frames to mostly horizontal in later frames. implying that some t.ype of temporal coherence constraint is necessary for correct nstimation of the flow from this image sequence. We have corrupted the images by adding an independent. Gaussian noise with a variance of ? to cach pixcl and then requantizing the resulting pixel values 10256 grey levels.

2. The fiow estimates and estimation crrors.

A $9 \times 9$ unit uniform stencil is used to spatially smooth the images hefore brightness gradients are computed. The computational paraments $\rho=400$ and $\nu=40$ have been used. Figure 2.4 shows frame 18 of the estimated flow vectors computed by the SF-ic and TCS-ic methods. The 


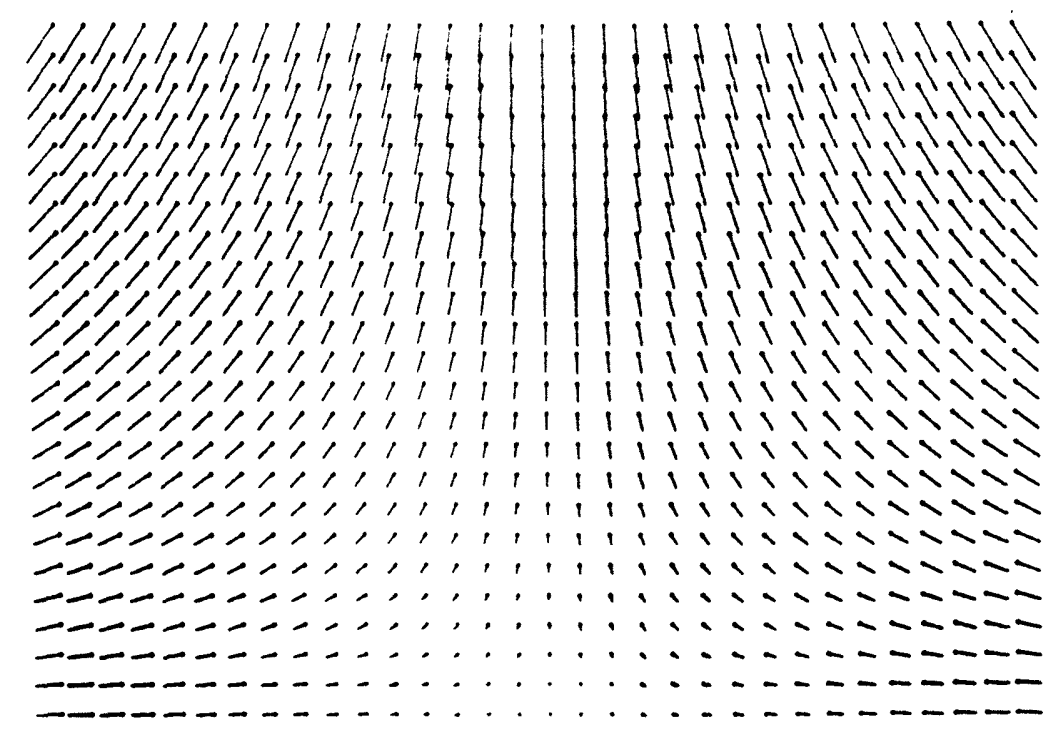

Finure 2.2: The true flow in the Stagnation Flow experiment. Every ot her flow irrtor along each axes is shown with a magnification factor of 4 for clarity.
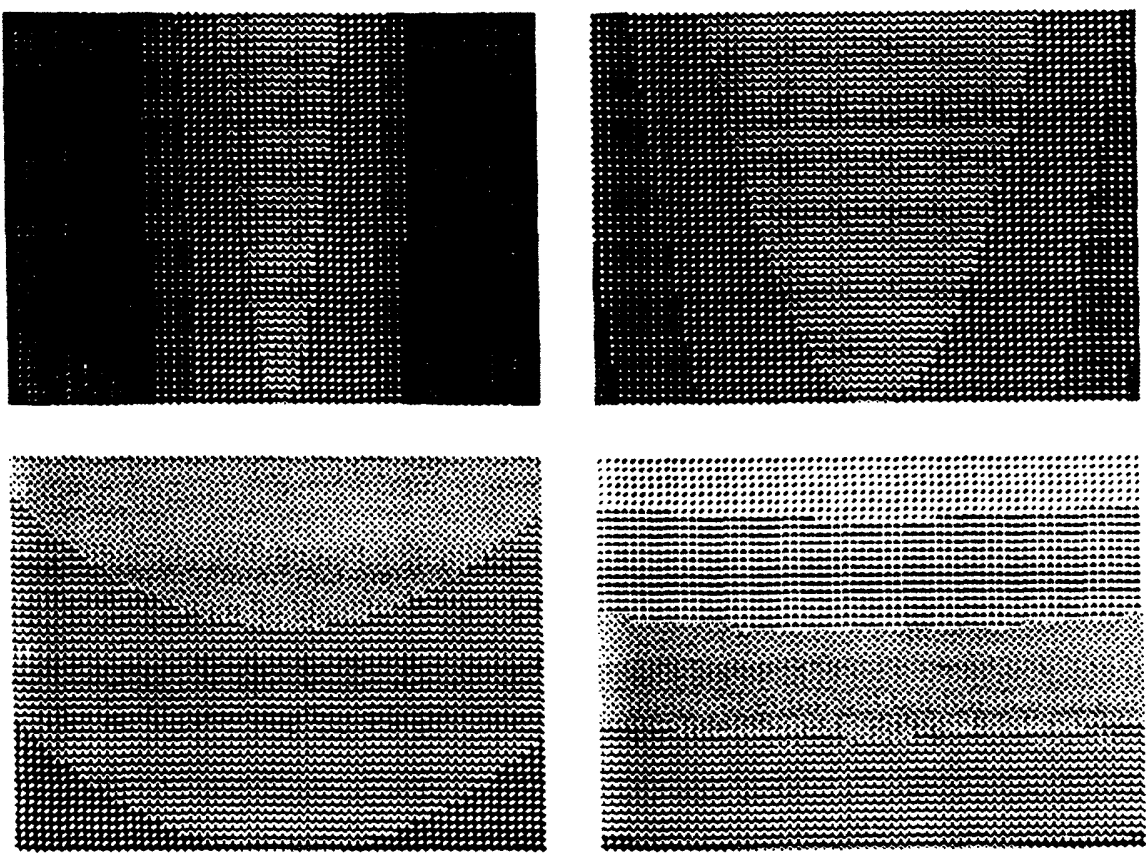

Fignre 2.3: The Stagnation Flow image sequence. Frames 0 and $i$ (top row) as wenl as 14 and 21 (bottom row) are shown. 


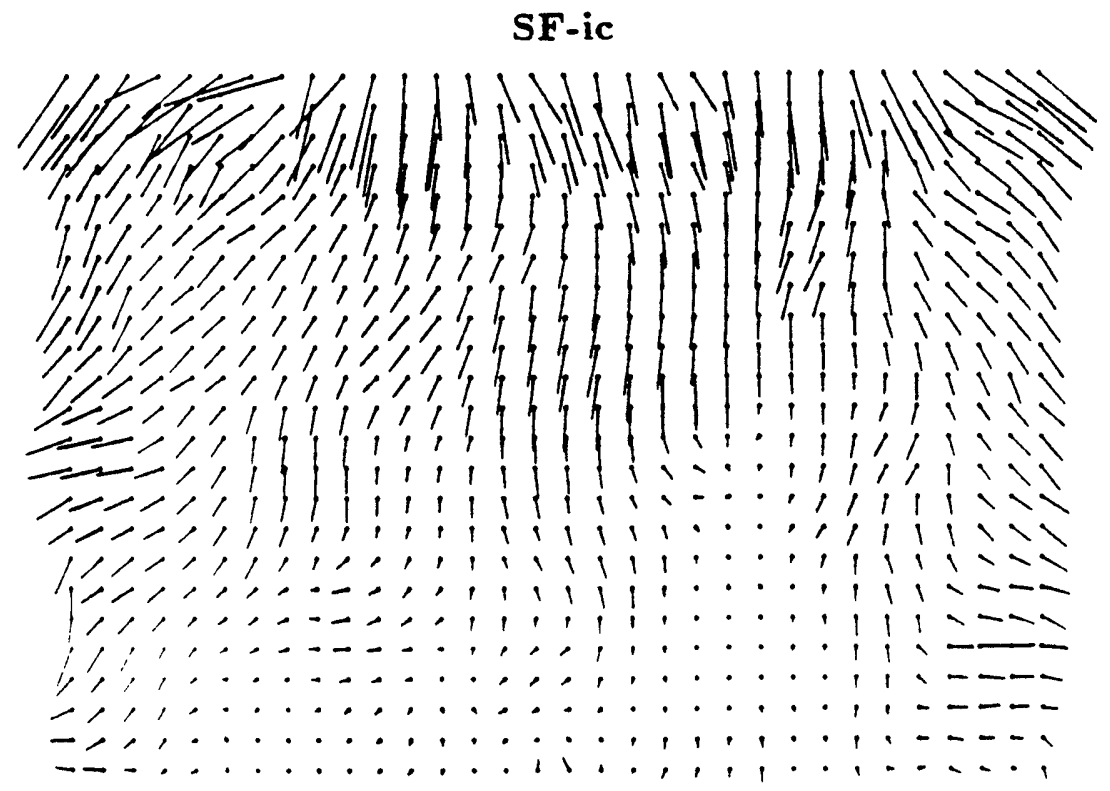

TCS-ic

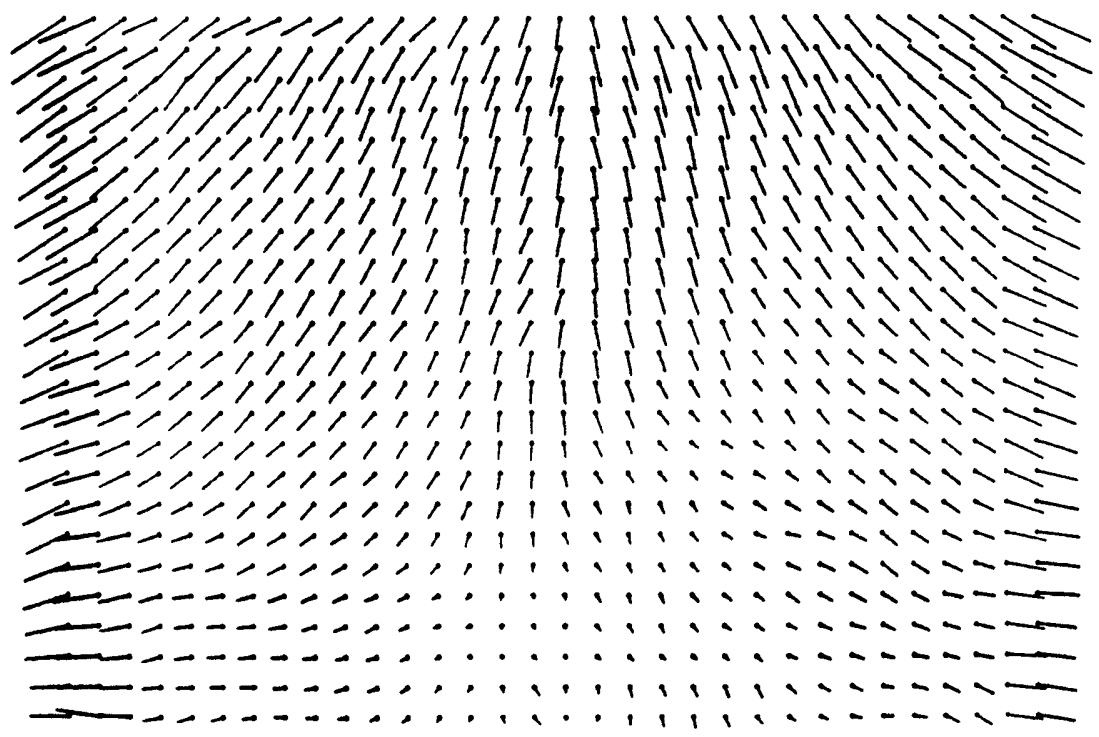

Figure 2.4: The optical flow estimates for frame 18 of the Stagnation Flow sequence by the SF-ic and TCS-ic methods. 
SF-ic method. without any provision for temporal data inlegration. has completely failed to reconstrurt the flow field. while the TCS-ic method has performed a reasonable reproduction of the flow in Figure 2.2. The llows computed by the SF-is and TCS-is are shown on Figure 2.5. which also displays the importance of temporal coherence in reconstruction. Figure 2.6 displavs the percent average estimation error for each $t$.

$$
\frac{\|\hat{\mathbf{x}}(t)-\mathbf{x}(t)\|^{2}}{\|\mathbf{x}(t)\|^{2}} \times 100
$$

where $\mathbf{x}(t)$ is the true flow and $\hat{\mathbf{x}}(t)$ is the estimated flow. for the four methods. These errors are consistent with our previous observations. Again. superior performance of the TCS-tvpe methods over the SF-type methods is displayed rather dramatically by the error curves.

\subsection{Multiscale Model-Based Estimation}

()IIr of the una jor computational bottlenecks of the halman filtering algorithm in tintion 2.4 is the spatial estimation problem represented by $(2.19)$. In section 2.1.1 we were able to transform this step of the multi-frame problem back In the level of complexity of the single-frame Horn and Schunk formulation as a resull. of our reduced-order approximation to the field model (2.21), which resulted in a sparse and banded structure of the matrix $\hat{L}(t)$. While such an ipproximation succeeds in making the multi-frame problem tractable while presurving near optimality of the resulting estimates and indeed represents. to nur kinnwlerlge. the first implementation of the complete Falman filtering equations in problems of this scale. the resulting inverse problem still leads to compuralinllally intensive algorithms. Specifically. the associated set of equations (2.19). while sparse. is extremely large. corresponding to discrete versions of elliptic partial differential equations [9]. The standard approaches to solving such large wis of linear equations, such as the Cianss-Seidel [9. 26]. multigrid [24]. and suc(msive ower-relaxation (SOR) [27] algorithms. are iterative. requiring increasing IIII!)hers of iterations (and thus increasing per pixel computational load) as the iminge size grows.

In this section we examine a novel approach to such large, computationally intrnsive spatial estimation problems wherein we combine a multiscale prior modrl of the field with a set of field observations. Recall from Section 2.4.1 that. Wr may view the update step of the optimal Kalman filter as such a static spatial estimation problem whercin a prior spatial ficld model (2.2:2) is combinod "ith a set of observations $g(t)$. The use of a multiscale modeling paradigm liarls to extremely efficient estimation algorithns which hold the promise of Irmmatirally reducing the computation required to solve such problems. For 'xample. the resulting multiscale algorithm is not iterative and in fact requires a fixed number of floating point operations per pixel independent of imagr sizr. Thus. the computational savings associated with the new approach actually inrreases as the image size grows. For simplicity and to illustrate the issies involved we will focus only on the single-frame case here. For clarity we will 


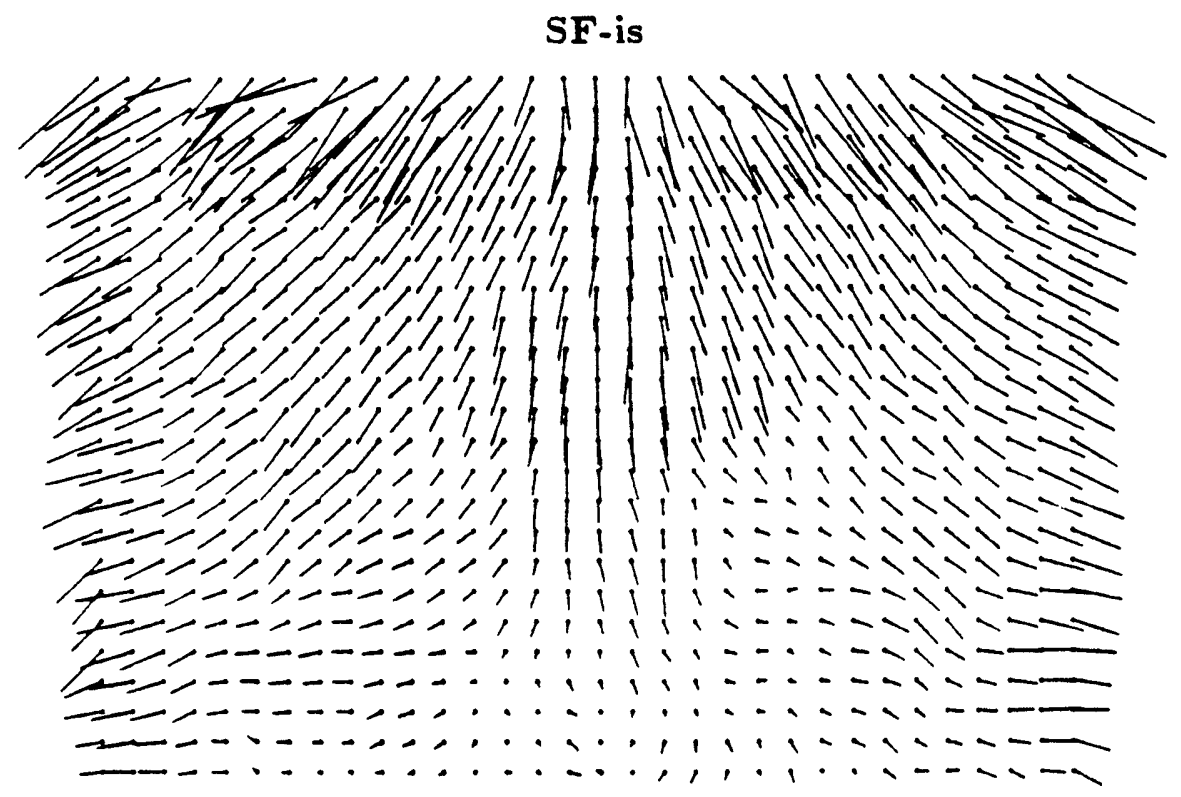

TCS-is

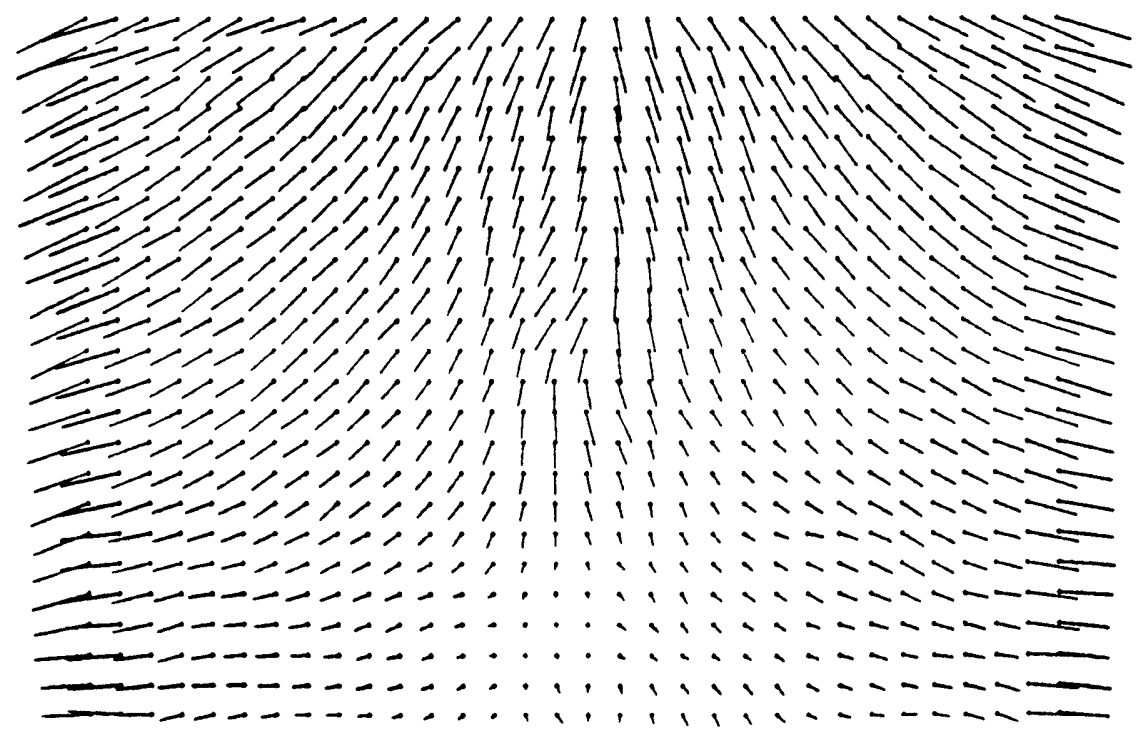

Figure 2.5: The optical flow estimates for the frame 18 of the Stagnation Flow sequence by the SF-is method and TCS-is method. 


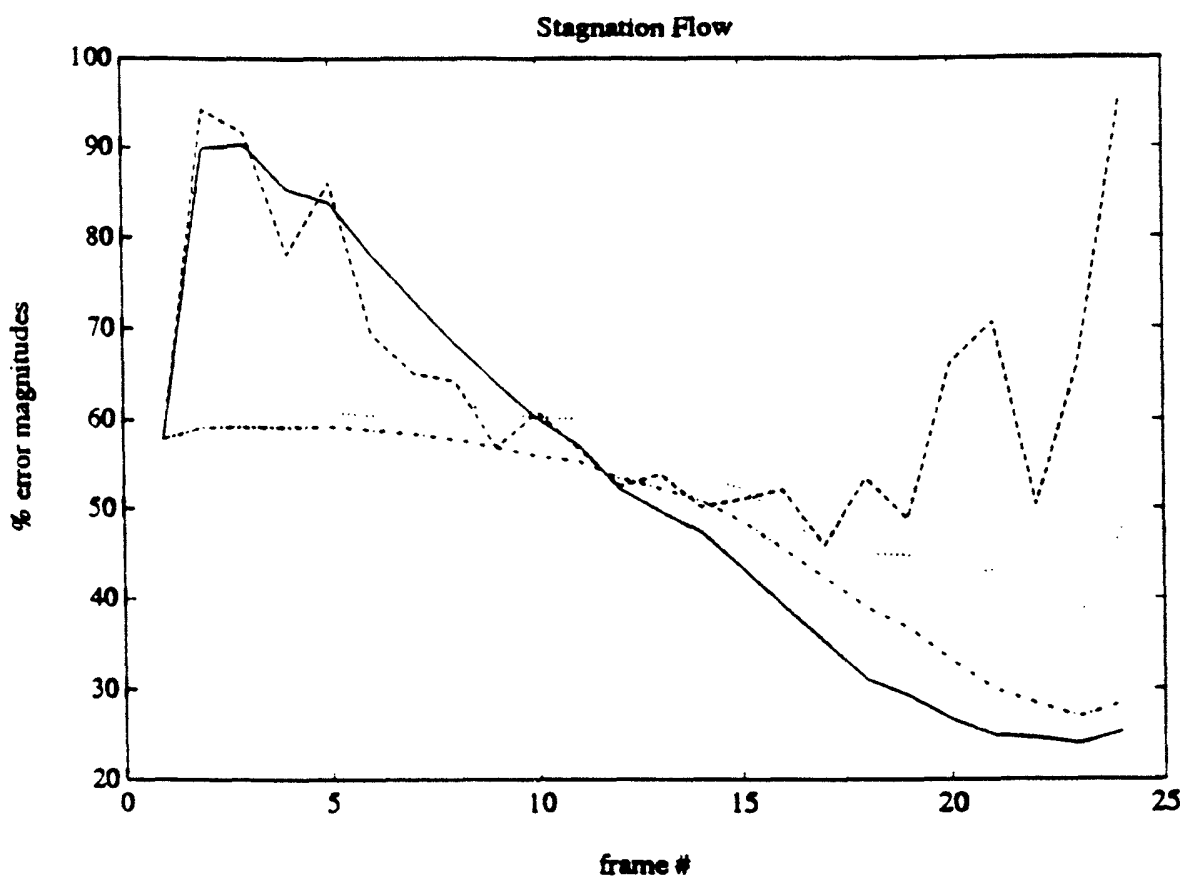

Fienre 2.(j: The estimation errors by the TCS-ic (solid-line). SF-ic (dashedlini.). SF-is (dotted-line), and TCS-is (dash-clot line) methods for the Stagnalion How experiment.

drop the lime index $t$ from the notation for the rest of this section. with the understanding that all quantities are taken at this point in time.

Recall. we argued in Section 2.3.1 that the single-frame optical flow estimalc's corresponding to $(2.5)$ conld be viewed as arising from the combination of lhe prinr statistical spatial model (2.8), corresponding to the smoothness contraint. and the observations (2.6). Now. there is nothing special about the prior morlel $(2.8)$ associated with the smoothness constraint. Thus we are lead to the idea of using a different class of prior models, capabable of capturing a wide mange of phenomenon. and in particular of yielding behavior which is similar in nature to that corresponding to the smoothness constraint. but which lead in computationally more attractive problem formulations. That is. we want to rhange lhe smoothness constraint term $x^{T} S^{T} \mathbf{S x}$ in $(2 . j)$ to snmething similar. salv. $\mathrm{x}^{T} \Lambda \mathrm{x} \approx \mathrm{x}^{T} \mathrm{~S}^{T} \mathrm{Sx}$ (where $\Lambda$ is a srmmetric positive semi-definite matrix) sinch that the resulting optimization problem is easy to solve. If we factor $\Lambda$ as $\Lambda=S^{T} \ddot{S}$ then we can interpret the new constraint as a prior probabilistic model just as we did with the smoothness constraint. In addition. there is a precise interpretation of what we have done as a Bayesian estimation problem. Sipreifically. if $\Lambda$ is invertible. then the use of this new constraint in place of the -moorhness constraint is equivalent to modeling the flow field probabilistically as $x \sim\left(0 . \Lambda^{-1}\right)$. since in this case the Bayes least squares estimate of the flow 
field $\mathbf{x}$. given this prior model and the measurements in $(2.6)$ is provided by:

$$
\dot{\mathbf{x}}_{B L S E}=\underset{\mathbf{x}}{\operatorname{argmin}}\left\{(\mathbf{y}-\mathbf{C x})^{T} \mathbf{W}(\mathbf{y}-\mathbf{C x})+\mathbf{x}^{T} \Lambda \mathbf{x}\right\}
$$

The normal equations corresponding to $(2.25)$ are given $b y$ :

$$
\left(\mathbf{C}^{T} \mathbf{W C}+\Lambda\right) \hat{\mathbf{x}}_{B L S E}=\mathbf{C}^{T} \mathbf{W} \mathbf{y}
$$

Comparison of the problem formulations $(2.5)$ and $(2.25)$, or of $t$ : tions $(2.9)$ and $(2.20)$. makes it apparent how the two problem $f$ the heart of the problem. We introduce our class of new models next.

\subsubsection{A Class of Multiscale Models}

The models we utilize to replace the smoothness constraint prior moci: were recenty introduced in $[28,29.30 .31]$. These models represent the flow fiedd at multiple acales. i.e. for a set of scales $m=0 \ldots \ldots, M$. with $m=0$ bering the coarsest scale and $m=M$ the finest scale. We define a set of optical flow fields indexed by scale and space. namely $x_{m}(i, j)$. At the m-th scale. the field consists of $t^{\prime \prime \prime}$ flow vectors. as illustrated in Figure 2.T. capturing features of the optical flow field discernible at that scale (i.e. finer-resolution features of the field appear only in finer-scale representations). Thus. the coarsest version of the flow field consists of just a single vector, corresponding to the average value of the optical flow over the entire spatial domain of interest. and successively finer versions consist of a geometrically increasing number of vectors. It the finest level. the flow field is represented on a grid with the same resolution as the image brightness data. In particular. $x_{M}(i . j)$ corresponds 10 the optical flow vector $x(i . j)$ in $(2 . t)$.

Abstractly, we are representing the flow field on the quadtres strusture illustrated in Figure 2.9. Pyramidal data structures such as the quadtrce naturally arise in image processing algorithms which have a multiscale component. For in. stance. sucressive filtering and decimation operations lead to images defincd on such a hierarchy of grids in the Laplacian pyramid coding algorithm of Burt and Adelson [32] and in the closely related wavelet transform decomposition of images [33]. Also. the multigrid approaches to low level vision problems discussed by Terzopoulos $[2 t]$ involve relaxation on a similar sequence of grids.

The model we introduce in this section describes in a probabilistic manner how the optical flow field $x(i, j)=r_{M}(i . j)$ is constructed by adding detail from one scale to the next. Just as the smoothness constraint prior model (2.s) describes probabilistic constraints among values of the optical flow at different spatial locations. our multiscale model describes such constraints among values at different scales. That is. our model describes the probabilistic evolution of $x_{m}(i . j)$ as the scale $m$ evolves from coarse to fine. For notational convenience in describing such models. We denote nodes on the quadt ree with a single abstract index $s$ which is associated with the 3 -tuple $(m . i . j)$ where again. $m$ is the scale and $(i, j)$ is a ripatial location in the grid at the m-rh seaie. It is alco nseful : 

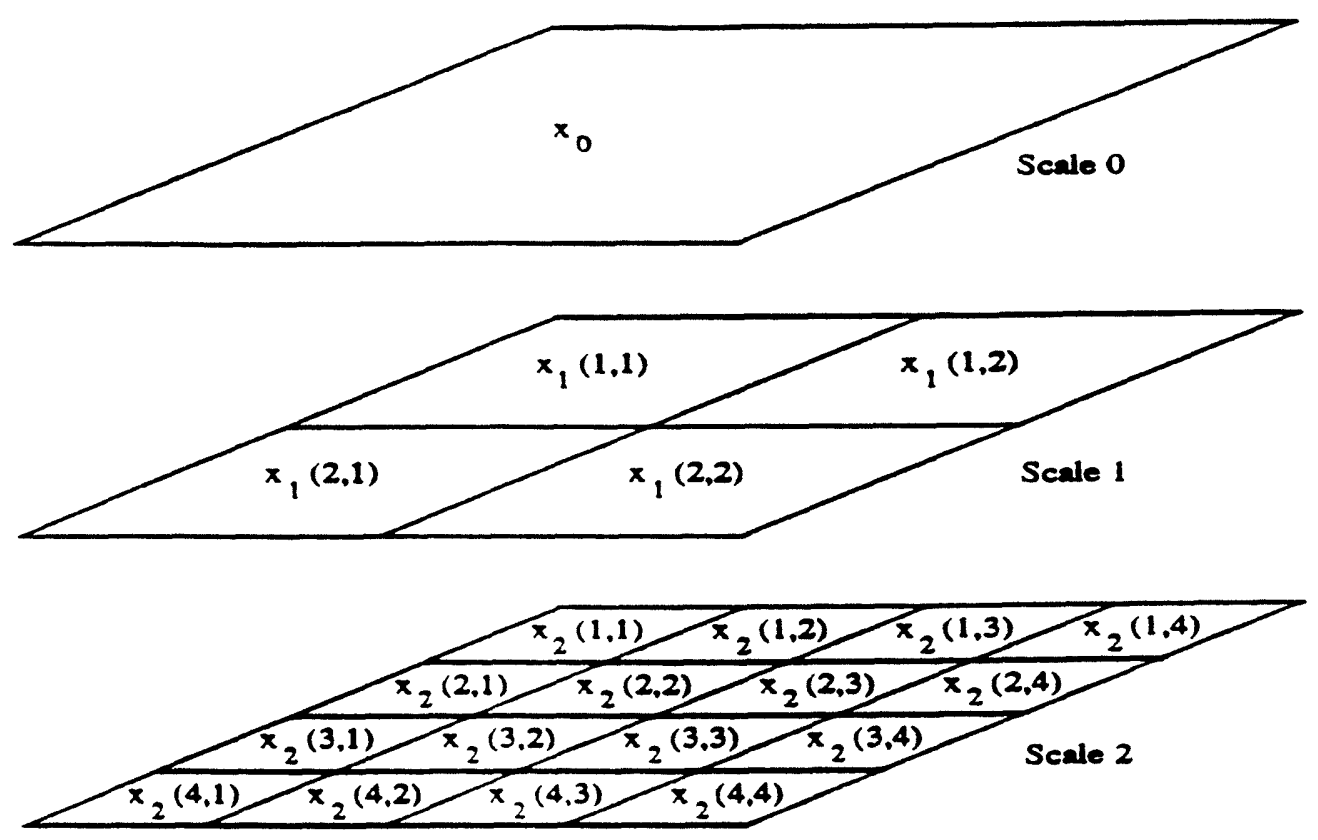

Figure 2.T: The structure of a multiscale optical flow field is depicted. The componculs of the ficld are denoted $x_{m}(i, j)$ where $m$ refers to the scale and the pair (i.j) denotes a particular grid location at a given scale. At the coarsest scalr. Hhere is a single flow vector and. more generally. at the m-th scale there are l'"' rectors.

deline an upurard shift operntor $\bar{y}$. In particular. the parent of node $s$ is denoted $\therefore$ (see Figure 2.8). We note that the operator $\bar{\gamma}$ is not one-to-one: it is in fact linir-1(n-one since each node will have four "offspring" at the next scale. For instance. if s corresponds to any of the nodes in the upper left quadrant of the scrond level grid (see Figure 2.T). i.e. nodes $(2.1 .1),(2.2 .1),(2.1 .2)$ or $(2.2 .2)$. then si corresponds to their parent on the first level. namely node (1.1.1).

Ifis are now in a position to define the class of multiscale models which lascribs the evolution of a multiscale stochastic processes indexed by nocles (11) the (luarltree. Specifically a stochastic quadtree process $x(s)$ is described rerritsively by:

$$
x(s)=A(s) x(s \bar{y})+B(s) u(s)
$$

Indere the following assumptions:

$$
\begin{aligned}
x_{0} & \sim\left(0 . P_{0}\right) \\
w(s) & \sim(0 . I)
\end{aligned}
$$

Thre rectors $r(s)$ and $u(s)$ are referred to as the state and driving noise terms. Thr slate variable $r_{0}$ at the root node of the tree provides an initial condition fin the recursion. The driving noise is white in both space and scale. and is Incorrelated with the initial condition. Interpreting each level as a representalion of a two-dimensional field. we see that $(2.27)$ describes the evolution of the 


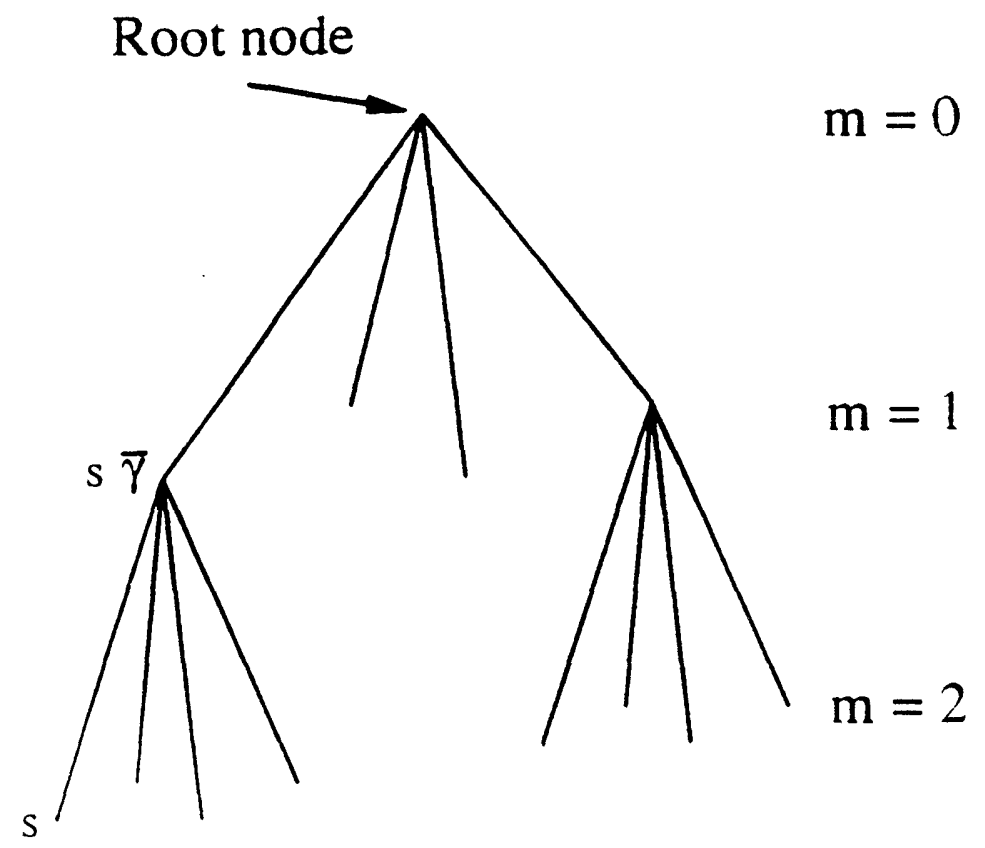

Figure 2.S: Qnadtree structure on which the multiscale proresses are defined. The abstract index $s$ refers to a node in the quadtree: $s \bar{y}$ refers to the parent of nodes.

process from coarse to fine scales. The term $A(s) x(s \bar{\gamma})$ represents interpolation down to the next level. and $B(s) w(s)$ represents higher resolution detail added as the process evolves from one scale to the next. In the application of interest here, $x(s)=x_{n}(i, j)$, where $s=(m, i . j)$, and thus $A . B \in K^{2 \times 2}$. Such a model corresponds in essence to a first-order recursion in scale for optical flow.

Measurements of the finest level optical flow field are available from the brightness constraint. In particular. at the grid point $(i, j)$ at the finest level $M$. we have a measurement equation corresponding to that in $(2.4)$ :

$$
y(i, j)=C^{\prime}(i, j) x_{M}(i, j)+v(i . j) \quad v(i, j) \sim\left(0 . v^{-1}(i . j)\right)
$$

where $C^{\prime}(i, j) \in \mathrm{H}^{1 \times 2}$ is the spatial brightness gradient at location $(i, j)$ and the white Ganssian observation noise is assumed to be independent of the initial condition $x, y$ and the driving noise $u(s)$ in $(2.2 \tau)-(2.29)$. Of course. We can group the state variables $x(s)$ at the finest level into a vector $\mathbf{x}_{M}$ as well as the corresponding measurements $y(s)$ and spatial gradient terms $C^{\prime}(s)$ in the same way as we did to get $(2.6)$ :

$$
\mathrm{y}=\mathrm{C} \mathrm{x}_{M}+\mathrm{v} \quad \mathrm{v} \sim\left(0 . \mathrm{W}^{-1}\right)
$$

We now have exactly the framework which led to the statement of $(2.2 .5)$ as an alternative to the smoothness constraint formulation (2.5). In particular.

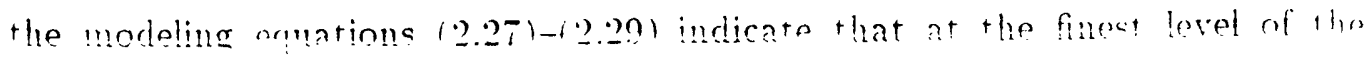


(juadtree. the flow field vectors will be a set of jointly Gaussian random variables $x_{M} \sim\left(0 . \Lambda^{-1}\right)$, where $\Lambda^{-1}$ is implicitly given by the parameters in $(2.27)-(2.29)$. The Bayes least squares estimate of $x_{M}$ given the measurements in $(2.31)$ and the prior model $(2.2 \tau)-(2.29)$ is then given by:

$$
\hat{\mathbf{x}}_{M I}=\underset{\mathbf{x}_{M}}{\operatorname{argmin}}\left(\mathrm{y}-C \mathbf{x}_{M}\right)^{T} \mathrm{~W}\left(\mathbf{y}-C \mathbf{x}_{M}\right)+\mathbf{x}_{M I}^{T} \Lambda \mathbf{x}_{M}
$$

The multiscale modeling framework thus provides an alternative to the smoothness constraint formulation of (2.5).

What remains to be done is to specify a model within this class that has (hancteristics similar to those of the smoothness constraint prior model. In particular. for our multiscale inodel based on $(2.2 T)-(2.29)$ to approximate the smonthness constraint prior we would like to choose our model parameters so Han. we have $\mathbf{S}^{T} \mathbf{S} \approx \Lambda$. The observation in Section 2.3.1 that the prior model $(2.8)$ implied by the operator $S$ in $(2.5)$ corresponds to a Brownian motion "fractal prior" suggests one approach to choosing the model parameters. In parlirular. the one-dimensional Brownian motion has a $1 / f^{2}$ generalized spectrum [अ2]. It has been demonstrated that such processes are well approximated by minlliscale models such as ours in one dimension if geometrically decreasing powirs of noise are added at each level $m$ of the process [30. 3.1]. In particular. this motivates the choice of $B(.9)=b t^{-\mu m(s)} I$ in $(2.2 i)$, where $b$ and $\mu$ are scalar constants. The constant $b$ directly controls the overall noise power in the process. .11sn. as discussed in [34]. the choice of $\mu$ controls the power law dependence of lhe generalized spectrum of the process at the finest resolution as well as the fractal dimension of its sample paths. Specifically. this spectrum has a $1 / f^{2 \mu}$ depmilence. Thus. the choice of $\mu=1$ would correspond to a Brownian-like fractal process. To achieve greater flexibility in both the modeling and estimalion. We allow $\mu$ to be a parameter that can be varied. In addition. recall that in the smoothness constraint formulation. $\mathbf{S}^{T} \mathbf{S}$ was not invertible because of the implicit assumption of infinite prior variance on the I)(: value of the optical flow lield. In our multiscale regularization context. this would correspond to setting llic intial covariance $P_{0}$ equal to infinity in $(2.28)$. This can be done without dilliculty in the estimation algorithms described next. but we have found that it is generally sufficient to simply choose $P_{0}$ to be a large multiple of the iclentity.

We have now specified a class of models which will allow us to approximate lire smoothess constraint prior model. The simple multiscale structure of these morlels leards to very efficient algorithms for computing the optimal estimate of Ilic state given a set of measurements. One of these algorithms. which we refer Io as the Multiscale Regularization (MR) algorithm. was devoloped in [28. 20. 311. 33i for one-dimensional signals. and its extension to images is described in $[3(i)$.

The MR algorithm computes the Baves least squares estimate of the state rortors $(2.27)$ given the measurements $(2.30)$ in two steps. The first step is iII "purard or fine-to-coarse sweep on the quadtree. Which propagates the marsurement information in parallel. level by level. from the fine scale nodes up to the root node. This step produces the best estimate at each node given all the data in the subtree under that node. At the top of the tree. one obtains the 
smoothed estimate of the root node. that is. the estimate of this node hased on all of the data. The smoothed estimate and associated error covariance at. the root node then provide intialization for the next step. This second step is a downward or coarse-to-fine sweep which propagates the global mea urement information now at the root node back down. and throughout the se. The result at. each node is the least squares estimate $\hat{x}^{s}(s)$ of the state b) based on all of the data. The resulting estimates at the finest level of tl: quadtree provide the solution to $(2.32)$. The resulting algorithm is just a gene, iization of the Rauch-Tung-Striebel smoothing algorithm [37] in scalf. The, ails of the upward and downward sweeps are discussed in greater detail in [30. 35. 36].

\subsubsection{Numerical Experiments}

Ilere we demonstrate the substantial computational benfit that can be achieved through the use of our multiscale modeling pardigm. 'To specify the MIR algorithm completely we need to choose the parameters of the model. We utilize the following parameterization:

$$
\begin{aligned}
r(s) & =r(s \bar{\gamma})+\left(b y^{-u m(s)}\right) u(s) \\
y(s) & =C^{\prime}(s) x(s)+v(s) \\
w(s) & \sim(0 . I) \\
v(s) & \sim\left(0 . v^{-1}(s)\right) \\
x_{0} & \sim(0 . p I)
\end{aligned}
$$

From (2.33) and (2.35) we see that the two components of the optical flow field are modeled as independent sets of random variables. and that each will haw a fractal-like characteristic dne to the choice of the driving noise gain $B(.8$ ) (as discussed in the previous section). We view $\mu$ and $b$ as free model parametors which can be varied to control the degree and type of regularization in much the same way that the parameter $\nu$ in the smoothness constraint formulation $(2.2)$ is used to tradeoff between the data dependent and regularization terms in the optimization functional.

As discussed previousty. the measurements $y(s)$ and measurement matrix $C(s)$ come directly from the image temporal and spatial gradients. which are available at the finest level of the quadtree. In the experiments clescribed below. We use a simple two-image difference to approximate the temporal gradient. The spatial gradient is computed by smoothing the image with a $3 \times 3$ Ciaussian kernel followed by a central difference approximation. The additive noise variance is given by $\nu^{-1}(s)$. We have found empirically that the choice $\nu^{-1}(s)=\max \left(\left\|C^{\prime}(s)\right\|^{2} .10\right)$ works well. This choice effectively penalizes large spatial gradients. which are likely points of occlusion where the hrightness constraint equation will not hold [38]. The parameter $p$ in the prior covariance of the root node was set to $p=100$. The distribution $(2.37)$ on the root node efiectively says that we are modeling the optical flow field components as zero mean random processes. The prior covariance reflects our confidence in this assumption. Since we do not believe that any prior assumption on the mean of optical flow field rnmponents can bo justificd. :- ont the paramerer $p$ such that 


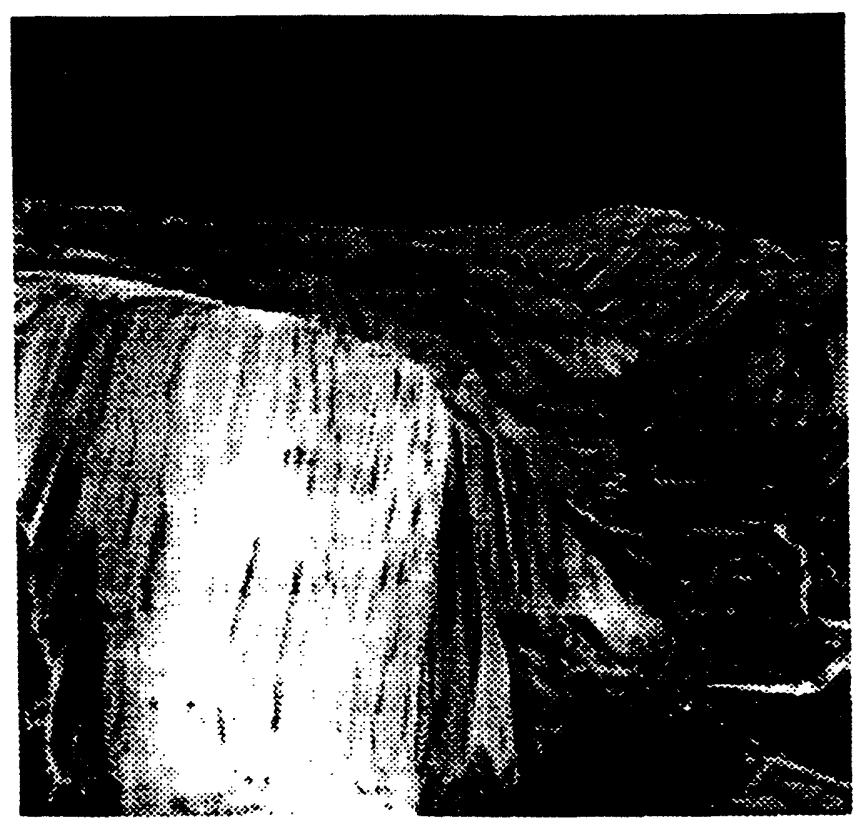

Figure 2.9: First trame of "Yosemite" sequence.

Hhe implied standard deviation is much larger than the sizes of the flow ficlds wre expect to see.

We compare our approach computationally and risually to the the (ianssSiridel ( $(i S)$ and successive over-plaxation (SOR) algorithms. which can be used in compute the solution of the smoothness constraint formulation given by (2.5). Silraight forward analysis shows that the CSS and SOR algorithms require 14 and is llonting point operations (flops) per pixel per iteration respectively. The number of iterations required for convergence of the itcrative aleorithms grows with image size [27]. For reasonable size images (say. $512 \times 512)$. SOR may mpinire on the order of hundreds of iterations to converge. so that the total compuntation per pixel can be on the order of $10^{3}-10^{4}$ nops. On the other hand. Ihe MIR algorithm requires if llops per pixel. Note that the MR algorithm is Int iterative. Thus. the computational gain associated with the MR algorithm an he on the order of one to two orders of magnitude. Details may be found in [3ii].

\section{Yosemite Sequence Experiment}

This example is a synthetic $256 \times 2.56$ image sequence which simulates the view whained by flying through the lusemite Valle ${ }^{2}$. The first inage in the sequence is shown in Figure 2.9 along with the actual flow field in Figure 2.10. The flow computed via the $\mathrm{UR}$ algorithm is shown in Figure 2.11 and the smoothnesin anstraint solution is shown in Figure 2.12. The smoothness constraint How

\footnotetext{
This sequence was syuthesized br irn () uam of SRI International.
} 


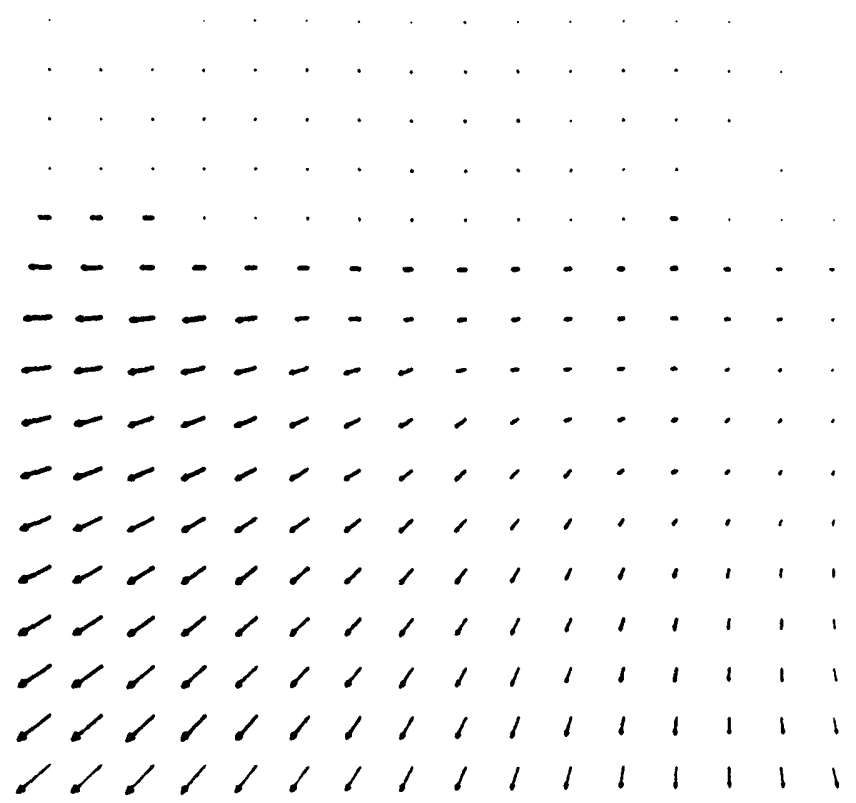

Figure 2.10: Yosemite sequence true optical flow.

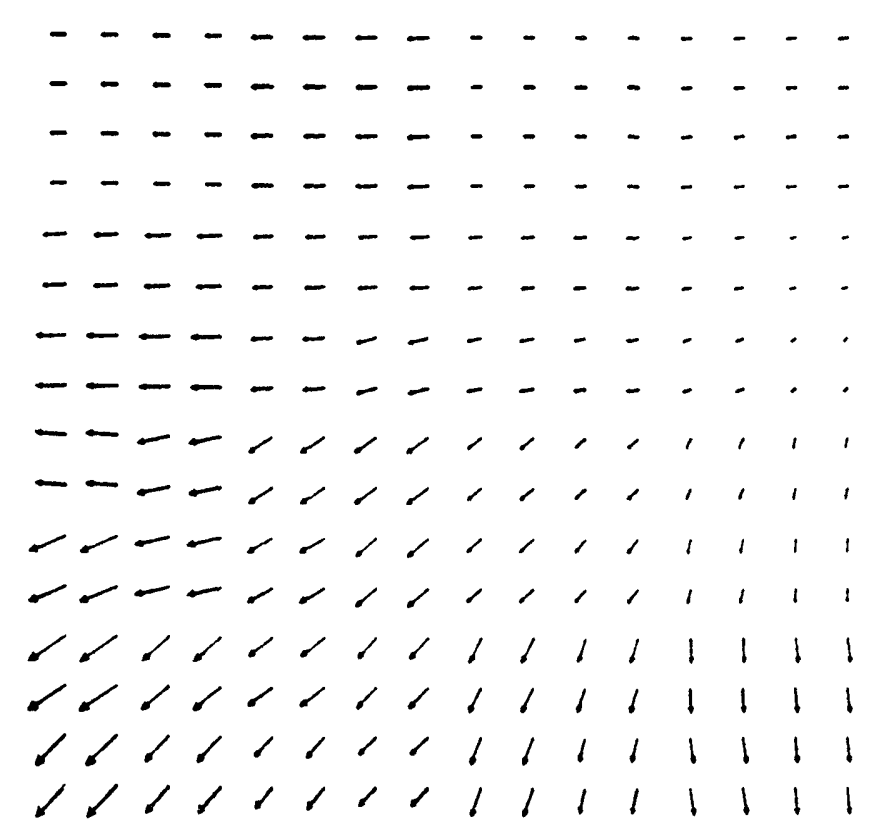

Figure 2.11: MIR algorithm flow estimates: $b=10 . \mu=2.5$. 


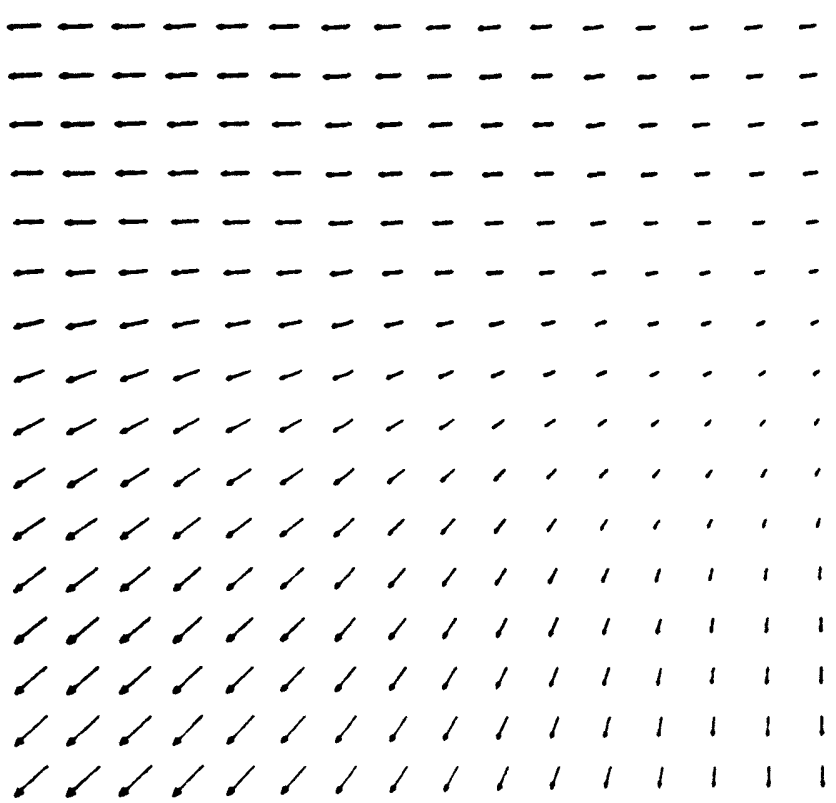

Fignre 2.12: SOR algorithm flow estimates: $R=500^{2} .250$ iterations. The SOR algorillum required a factor of 60 more computation in this example.

mimales required 250 SOR itcrations in this example. representing a factor of 60 morn computation than the MR estimates. Note the substantial increase over the previons example in the number of iterations required for the SOR algorithm to inlierge. The number of iterations required for convergence depends on several llings. including the parameter $\nu$, the image gradient characteristics and the image size. Theoretical analysis in [2i] shows that the SOR algorithm requires 'in the order of $n$ iterations for an $n \times n$ image. Thus. we expect substantially mom romputational savings as the image size increases.

I root mean square (rms) error comparison of the algorithms is shown in linnre 2.13. As expected. the SOR algorithm is significantly faster than the (is) algorithm (they will converge to the same result since they are solving the ame partial differential equation). The rms error in the MR flow estimates is depicted as a straight line. since the algorithm is not iterative. Neither of the istimales coincides with the actual optical flow. but they do have comparable rms rror as in the previous example. In addition. the figure illustrates the rnupulational advantage of the MIR algorithm. In particular. the SOR algorithm is still reducing the rums error in its How estimates after 300 iterations. at which point the IIR algorithm requires a factor of $300 / 4.2=i 1.4$ less computation.

This image sequence contains a problem often encountered in real images: reuions of constant intensity. The problem is that the lack of gradient information in that region implies that the optical flow is not well defined. The smoothness constraint and multiscale prior models provide a means of interpolating out into lhese regions. The result of this is apparent in the top portion of Figures 2.11 


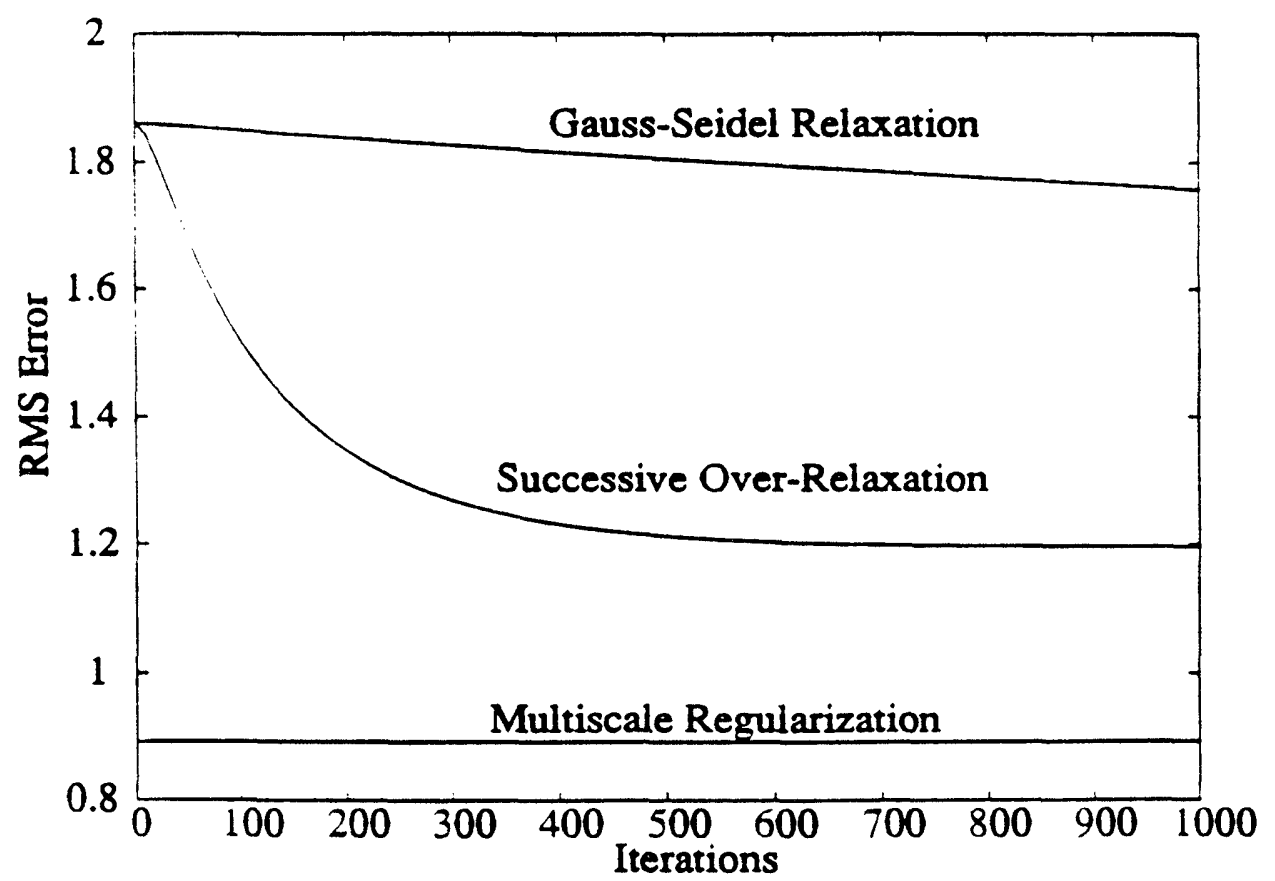

Figure 2.13: Rms Error Comparison of MR. SOR and Ciauss-Seidel ( CiS') algorithm flow estimates for the Yosemite sequence. 
and 2.12. In advantage of the IIR formulation is that it accomplishes this "xrmpolation at an appropriately coarser. and hence computationally simpler. seales.

Vote the the MR and $S($ ' How estimates are not identical due to difrerences in the prior models. If there is particular interest in ohtaining the $S C^{\prime}$ ' solution. the clinstion arises of using the MR solution as an initial guess for the iterative algorithms which compute the $S C^{\prime}$ 'solution. Note that the difference between the St and MR llow estimates is associated with the non-smooth. high frequency aspects of the MIR flow at block edges. It is precisely these high frequency components that are quickly removed by SOR or (iS algorithms computing the the smoothness constraint solution and suggests that the MIR algorithm wonld provide an excellent pre-ronditioner for the iterative algorithms.

\subsection{Conclusions}

IVi have taken an estimation-theoretic perspective to image sequence processing using as nur whicle the computation of optical flow. ()ur results made use of the interprention of various components of standard formulations of this problem as stalisticnl modkls. First we presented a near-optimal Kalman filter for the "stimation of optical flow under a temporal colerence constraint and hased on the propagation of approximate local models of the estimation error covariance. This filter provides. to our knowledge. the first implementation of the romplets lialman filtering equations for space-time problems of this scale. and the only riampife of successful. near optimal. propagation of covariance matrices of this iize.

Vext we used the observation that both the single and multi-frame problems an br formulated as spatial estimation problems. wherein sets of nbserwations arr fused with prior spatial field models. to motivate the use of a recently deinloperl rlass of muitisents statistical modols in their solution. The algorithms arising from this class of models allows dramatir speedups in computational - mend. Por cimplicity we enncentrated on the single frame case. hough we conld ilso 11 s for the static spatial estimation problem occuring in the multi-frame silnal ion. I particularly interesting and open question is how 10 directly propagate nnc of the multiscale models used in Section 2.5 in time.

\section{Acknowledgements}

This work was partially sllpported hy the ('harlos Stark Draper Taboratory IR., 1) Program under (irant DL-II-418524. the Office of Naral Research under

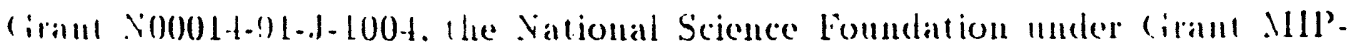

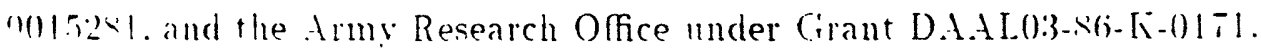




\section{Bibliography}

[1] A. Mevgret and M. Thonnat. "Segmentation of optical flow and 3D data for the interpretation of mobile objects." in Third International ('onference on Computer lision. pp. 2:38-245. IEEE C'omputer Society Press. 1990. ()saka. Japan.

[2] F. Heitz. P. Perez. E. Memin. and P. Bouthemy, "Parallel visual motion analysis using multiscale Markov random fields." in Procecdings of Itorkshop on lisual Motion. IEEE ('omputer Society Press. 1991. Princeton. V.J.

[3] II. ('. Tonguet-Htiggins and $K$. Prazdny, "The interpretation of a moving rolinal image." Procedings of the Royal Society of London. B. vol. 20 S. Pp. 38.5-397. 1980.

[1] S. Negahdariponr and B. K. IIorn. "Jirect passive navigation." IEEE Tinnsartions on Pattem Analysis and Machine Intelligcnce. vol. PAMI-9. 1987.

[5] N. Baaziz and C'. Labit. "Multigrid motion estimation on pyramidal representations for image sequence coding," Tech. Rep. 5i2. IRISA. Feb. 1991.

[i] D. Walker and K. Rao. "Improved pei-recursive motion compensation." IEEE Transactions on ('ommunications. vol. 32. pp. 1128-1134. 1984.

[i] .1. Rongee. B. ('. Lery. and A. S. Willsky. ". In estimation-based approach to the reconstruction of optical flow." Tech. Rep. LIDS-P-1663. Laboratory for Information and Decision Systems. Massachusetts Institute of Technology. 1987.

[i] . A. Rougee. B. Levy. and A. S. Willsky. "Reconstruction of two dimensional velocity fields as a linear estimation problem." in Procefdings lst Internatimnal C'onference on Computer lïsion. (London. England). pp. (5+6-6.50. $198 T$.

[9] B. K. P. Horn and B. ('. Schunck. "Determining optical flow." Irtificinl Intelligenre. vol. 17. pp. 185-203. 1981.

[10] M. J. Black and P. Anandan. "A model for the detection of motion over time." in Third Intcrnational C'onference on C'omputer lïsion. pp. 333-3i. IEEE ('omputer Society Press. 1990. Osaka. Japan. 
[11] A. Singh. "Incremental estimation of image-flow using a Kalman filter."

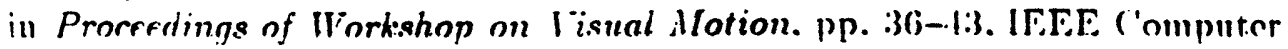
Society Press. 1991. Princetoll. N.J.

[12] R. Szeliski. Bnysian Modeling of Incertainty in Lou-level Tision. Norwell. Massachuesetts: Kluwer Academic Publishers, 1989.

[13] B. D. O. Anderson and J. B. Moore. Optimal Filtcring. Englewood C'liffs. N.J.: Prentice-Hall. 1979.

[14] A. Cielb. ed.. Ipplifd Optimal Estimation. C'ambridge. MA: MIT Press. 1074.

[15] F. L. Lewis. Optimal Estimation. New York: John Wiley d: Sons. 1986.

[16] T. M. ('hin. W. C. Karl. and A. S. Willsky. "Sequential filtering for multiframe visual reconstruction." to appear in Signul Processing. Aug. 1992.

[1i] M. Bertero. T. Poggio. and V. Torre. "Ill-posed problems in early vision." Procredings of the IEEE. vol. i(i. pp. R(69)-8\$9. 1989.

[18] E. ( $\therefore$ Ilildreth. "Computations underlying the measurement of risual motion." Irtificial Intclligence, vol. 2:3. pp. 309-35.4. 195.t.

[19] N. M. Cirzywacz. J. A. Smith. and A. L. Yuille. *A common theoretical framework for visual motion's spatial and temporal coherence." in Prorerrlings of I'orkishop on I isual Motinn. pp. 149-155. IEEE ('omputer Society Press. 1989. Irvine. C.A.

[20] L. H. Matthies. R. Szeliski. and T. Kanade. "Kalman filter-based algorithms for estimating depth from image sequences." Intrrnational Journal of Computer lïsion. vol. 3. 1989.

[21] T. .1. ('hin. Dynamis. Estimation in C'omputational lission. PhD lhesin. Massachusetts Institute of Technologr. 1991.

[22] B. Mandidbrot and II. V. Ness. "Fractional Brownian motions. fractional noises and applications." SIAM Revicu. vol. 10. pp. 422-4363. 1968.

[23] B. (. Levr. .I. B. Adams. and A. S. Willsky. "Solution and linear estimation of 2-D nearest-neighbor models." Proceedings of the IEEE. vol. 7s. pp. 62 i(i) 1.990.

[2.1] D. Terzopoulos. "Image analysis using multigrid rolaxation modrls." IF:H:

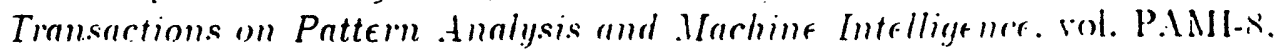
pp. 129-139. 1986.

[25] M. ('. Potter and J. F. Foss. Fluid Merhanirs. Okemos. Michigan: Cireat Lakes Press. 1982.

[26] C:. Strang. Introduction to Applied Mathrmatirs. Wellesley. MA: Vellesley('ambridge Press. 1986. 
[27] (… ... Kuo. B. C . Levy, and B. R. Musicus. "A local relaxation method for solving elliptic PDF's on mesh connected arravs." STAM J. S'ici. S'tat. ('omput.. vol. 8. pp. 550-573. 1987.

[2R] K. C'. ('hou. A. S. Willsky, A. Benveniste, and M. Basseville. "-Recursive and iterative estimation algorithms for multiresolution stochastic processes." in Pror. of the IEEE Conference on Decision and Control. Dec. 1989.

[29] K. ('. ('hou. A stochastic modeling approach to multiscale signal processing. PhD thesis. Massachusetts Institute of Technology, 1991.

[30] K. ('. C'hou. A. S. Willsky, and A. Benveniste. "Multiscale recursive estimation. data fusion and regularization." submitted to IEEE Transactions on Automatic C'ontrol. 1992.

[31] S. ('. ('lippingdale and R. C. Wilson. "Least squares image estimation on a multiresolution pyramid." in Proc. of the 1989 International C'onferenre on Acoustics. Speech. and Signal Processing, 1989.

[32] P. Burt and E. Adelson. "The Laplacian Pyramid as a compact image code." IEEE Transactions on Communications. vol. 31. pp. 482-540, 19\$3.

[333] S. Mallat. "Nulti-frequency channel decomposition of images and wavelet models." IEEE Transactions on .Icoustics. Specch. and Signal Processing, vol. 3i. pp. 2091-2110, 1989.

[3.1] (i. Wornell. "A Karhunen-Loeve like expansion for 1/f processes." IEEE Transactions on Information Theorey. vol. 36. pp. 8.59-861. 1990.

[35)] K. ('. ('hou. A. S. Willsky. and R. Nikoukhah. "Multiscale systems. Kalman filters and Riccati equations." submitted to IEEE Transactions on Automatic ('ontrol. 1992.

[36i] M. R. Luettgen. W. C'. Karl. and A. S. Willsky. "Optical flow computation vin multiscale regularization." submitted to IEEE Transactions on Image Processing. 1992.

[3i] II. E. Rauch. F. Tung, and C. T. Striebel. "Maximum likelihood estimates of linear dynamic systems." AHA Journal. vol. 3. pp. 14+5-1450. 1965.

[38] E. Simoncelli. E. Adelson. and D. Heeger. "Probability distributions of optical flow." in Proceedings of the IEEE C'onferrnce on C'omputer lïsion and Pattom Recoynition. (Maui. Ilawaii). June 1991. 